\title{
PERSISTENCE OF GAUSSIAN PROCESSES: NON-SUMMABLE CORRELATIONS
}

\author{
AMIR DEMBO* AND SUMIT MUKHERJEE
}

\begin{abstract}
Suppose the auto-correlations of real-valued, centered Gaussian process $Z(\cdot)$ are nonnegative and decay as $\rho(|s-t|)$ for some $\rho(\cdot)$ regularly varying at infinity of order $-\alpha \in[-1,0)$. With $I_{\rho}(t)=\int_{0}^{t} \rho(s) d s$ its primitive, we show that the persistence probabilities decay rate of $-\log \mathbb{P}\left(\sup _{t \in[0, T]}\{Z(t)\}<0\right)$ is precisely of order $\left(T / I_{\rho}(T)\right) \log I_{\rho}(T)$, thereby closing the gap between the lower and upper bounds of [NR], which stood as such for over fifty years. We demonstrate its usefulness by sharpening recent results of [Sak about the dependence on $d$ of such persistence decay for the Langevin dynamics of certain $\nabla \phi$-interface models on $\mathbb{Z}^{d}$.
\end{abstract}

\section{INTRODUCTION}

Persistence probabilities, namely the asymptotic of $\mathbb{P}\left(\sup _{t \in[0, T]}\{Z(t)\}<0\right)$ as $T \rightarrow \infty$, have a fairly long history in probability theory with the case of stationary, centered, Gaussian processes $Z(t)$ receiving much attention (c.f. [Sle, $\mathrm{NR}$, Shu, Wat, Pic, SM, DPSZ, Mol, AS] and the references therein). In particular, for non-negative, stationary auto-correlation $A(s, t)=\mathbb{E}[Z(s) Z(t)]$, it directly follows by an application of Slepian's lemma and sub-additivity that the limit

$$
b(A):=-\lim _{T \rightarrow \infty} \frac{1}{T} \log \mathbb{P}\left(\sup _{t \in[0, T]}\{Z(t)\}<0\right)
$$

exists in $[0, \infty]$, and it is easy to see that $b(A)$ is finite whenever $Z(\cdot)$ has continuous sample paths (c.f. Lemma 2.6). Hereafter for any stationary non-negative correlation function $A(\cdot, \cdot)$ we use the notation $b(A)$ to denote the limit defined in (1.1).

For such processes the positivity of $b(A)$, namely the exponential decay of the corresponding persistence probabilities, is equivalent to integrability of $A(0, \cdot)$, under certain regularity condition on $\tau \mapsto A(0, \tau)$.

For many processes of interest $\tau \mapsto A(s, s+\tau)$ is non-integrable, with $b(A)=0$ (see for example Remark 1.4, Corollary 1.10 and Remark 1.12). In such cases (1.1) is of limited value, and the finer, sub-exponential persistence probability decay rate, is of much interest. Indeed, focusing on the special case where $A(s, t)$ decays as $|t-s|^{-\alpha}$ for some $\alpha \in(0,1]$, already in 1962, Newell and Rosenblatt NR showed that

$$
\begin{aligned}
& T^{\alpha} \lesssim-\log \mathbb{P}\left(\sup _{t \in[0, T]}\{Z(t)\}<0\right) \lesssim T^{\alpha} \log T, \quad \text { for } \quad 0<\alpha<1, \\
& \frac{T}{\log T} \lesssim-\log \mathbb{P}\left(\sup _{t \in[0, T]}\{Z(t)\}<0\right) \lesssim T, \quad \text { for } \quad \alpha=1 \text {. }
\end{aligned}
$$

Date: September 12, 2016.

2010 Mathematics Subject Classification. Primary 60G15; Secondary 82C24.

Key words and phrases. Persistence probabilities, Gaussian processes, regularly varying, $\nabla \phi$-interface.

${ }^{*}$ Research partially supported by NSF grant DMS-1106627. 
Hereafter, for any non-negative functions $a_{1}(T), a_{2}(T)$, we denote by $a_{1}(T) \lesssim a_{2}(T)$ the existence of $C<\infty$, possibly depending on the law of $\{Z(\cdot)\}$, such that $a_{1}(T) \leq C a_{2}(T)$ for all $T$ large enough, with $a_{1}(T)=\Theta\left(a_{2}(T)\right)$ when both $a_{1}(T) \lesssim a_{2}(T)$ and $a_{2}(T) \lesssim a_{1}(T)$.

To the best of our knowledge, the gap between the upper and lower bounds of [NR], as in (1.2)(1.3), has never been improved. Our main result closes this gap, by determining the correct decay rate of the relevant persistence probabilities, in case of asymptotically stationary non-negative $A(s, t)$ that are regularly decaying in $|t-s|$ large. To this end, we shall make use of the following definition.

Definition 1.1. For $\alpha \geq 0$, let $\mathcal{R}_{\alpha}$ denote the collection of measurable, regularly varying of order $-\alpha$ functions $\rho:[0, \infty) \mapsto(0,1]$, i.e. for every $\lambda>0$ one has

$$
\lim _{t \rightarrow \infty} \frac{\rho(\lambda t)}{\rho(t)}=\lambda^{-\alpha}
$$

Associate to each $\rho \in \mathcal{R}_{\alpha}$ the primitive function $I_{\rho}:(0, \infty) \mapsto(0, \infty)$ such that

$$
I_{\rho}(t):=\int_{0}^{t} \rho(s) d s,
$$

and the asymptotic persistence decay rate

$$
a_{\rho}(t):=\frac{t \log I_{\rho}(t)}{I_{\rho}(t)} .
$$

Theorem 1.2. Suppose the centered Gaussian process $\{Z(t)\}_{t \geq 0}$ has non-negative auto-correlation

$$
A(s, t):=\frac{\mathbb{E}\left[Z_{s} Z_{t}\right]}{\sqrt{\mathbb{E}\left[Z_{s}^{2}\right] \mathbb{E}\left[Z_{t}^{2}\right]}}
$$

(a) If some $\alpha \in(0,1]$ and $\rho \in \mathcal{R}_{\alpha}$ with $I_{\rho}(\infty)=\infty$, are such that there exists $\widetilde{\eta}>0$ satisfying

$$
\limsup _{t, \tau \rightarrow \infty, \tau \leq \widetilde{\eta} t} \frac{A(t, t+\tau)}{\rho(\tau)}<\infty
$$

then,

$$
-\limsup _{T \rightarrow \infty} \frac{1}{a_{\rho}(T)} \log \mathbb{P}\left(\sup _{t \in[0, T]}\{Z(t)\}<0\right)>0 .
$$

(b) Suppose further that

$$
\lim _{u \downarrow 0} \sup _{s \geq 0} \mathbb{E}\left[\sup _{t \in[s, s+u]}\{Z(t)\}\right]<\infty,
$$

and there exists $\eta>0$ such that

$$
\liminf _{t, \tau \rightarrow \infty, \tau \leq \eta t} \frac{A(t, t+\tau)}{\rho(\tau)}>0 .
$$

Then,

$$
-\log \mathbb{P}\left(\sup _{t \in[0, T]}\{Z(t)\}<0\right)=\Theta\left(a_{\rho}(T)\right) .
$$


Theorem 1.2 is proved in Section 2, where for the upper bound of part (a) it suffices to consider the persistence probabilities over $[r T, T]$ for suitably chosen $r \in(0,1)$. We can further split $[r T, T]$ into sub-intervals while leaving large enough gaps to ensure that the dependence between the restrictions of $Z(t)$ to the different sub-intervals, is weak enough for deducing an exponential decay of the overall persistence probability in terms of the number of such sub-intervals. The more delicate proof of the complementary lower bound of part (b) consists of four steps. We first rely on Slepian's lemma and the non-negativity of the correlation $A(\cdot, \cdot)$ of (1.6) to show that if such lower bound holds for intervals $\left[r T^{\prime}, T^{\prime}\right]$ with $r \in(0,1)$ fixed and $T^{\prime}$ large enough, then it must also extend to the interval $[0, T]$. To verify such a bound for $[r T, T]$, in the second step we split it to many sub-intervals, now employing a conditioning argument to control the height of the end-points of these sub-intervals, provided that the conditioned process has non-negative correlations. The third step establishes the latter crucial fact, thanks to certain properties of any such correlation function, the derivations of which are deferred to the last step of the proof.

Remark 1.3. For $\alpha \in(0,1)$, upon comparing (1.5) and (2.1) we see that $a_{\rho}(T)=\Theta\left(\rho(T)^{-1} \log T\right)$. So, in this case our conclusion (1.11) is that the persistence probability lower bound of [NR], namely the RHS of (1.2), is tight. In contrast, $I_{\rho}(T)$ is not $\Theta(T \rho(T))$ when $\alpha=1$, and in particular $\rho(t)=1 /(1+t)$ yields $a_{\rho}(T)=T(\log \log T) /(\log T)$, with neither the upper nor the lower persistence probability bound of [NR] then tight.

Remark 1.4. The conclusion (1.11) holds for any stationary process $\{Z(\cdot)\}$ having non-negative auto-correlation $A(0, \tau)=\Theta(\rho(\tau))$ for some $\rho \in \mathcal{R}_{\alpha}, \alpha \in(0,1]$, such that $I_{\rho}(\infty)=\infty$ and $t \mapsto Z(t)$ has a.s. continuous sample path (which holds for example when $|\log u|^{\eta}(1-A(0, u)) \rightarrow 0$ as $u \rightarrow 0$, for some $\eta>1$, see [AT, (1.4.3)]). Such stationary Gaussian processes of algebraically decaying, non-summable correlations appear frequently in the physics literature (see for example [GPS, MB1, MB2, and the excellent survey in [BMS]). An interesting open problem is to find in this context sufficient conditions for the existence of the limit

$$
b_{*}(A):=-\lim _{T \rightarrow \infty} \frac{1}{a_{\rho}(T)} \log \mathbb{P}\left(\sup _{t \in[0, T]}\{Z(t)\}<0\right),
$$

possibly after replacing $a_{\rho}(T)$ of (1.5) by an equivalent function.

For slowly varying, eventually decaying to zero, correlations (namely, as in Theorem 1.2, but with $\rho \in \mathcal{R}_{0}$ ), we next determine the rate of decay of persistence probabilities, up to a log factor.

Proposition 1.5. Suppose in the setting of Theorem 1.2 that conditions (1.7), (1.9) and (1.10) hold for some $\rho \in \mathcal{R}_{0}$ which is eventually non-increasing and

$$
\lim _{x \rightarrow \infty} \rho(x)=0 \text {. }
$$

Then, we have that

$$
a_{\rho}(T) \lesssim-\log \mathbb{P}\left(\sup _{t \in[0, T]}\{Z(t)\}<0\right) \lesssim a_{\rho}(T) \log T .
$$

Remark 1.6. Recall that the spectral measure $\mu_{A}$ of a centered, stationary Gaussian process $\{Z(\cdot)\}$ is the unique non-negative measure such that

$$
A(0, \tau)=\int_{\mathbb{R}} e^{-i \lambda \tau} d \mu_{A}(\lambda) \quad \forall \tau \in \mathbb{R}
$$

and in particular, the absolute integrability of $A(0, \cdot)$ implies the existence of uniformly bounded density of $\mu_{A}$. Following [DV] treatment of discrete time, centered, stationary Gaussian sequences, $\left[\mathrm{BD}\right.$, Theorem 2.1] derives the Large Deviations Principle (LDP) at speed $T$ and $C_{b}(\mathbb{R})$-topology, for 
$L_{T}:=T^{-1} \int_{0}^{T} \delta_{Z(t)} d t$, provided $\mu_{A}$ has a vanishing at infinity, continuous density. This does not imply the LDP for $L_{T}(-\infty, 0)$, so when going beyond non-negative correlations, the limit in (1.1) might not exist. Nevertheless, $[\mathrm{FF}$ ] provide in this setting sufficient conditions for truly exponential decay of the persistence probabilities. Specifically, [FF] shows that

$$
-\log \mathbb{P}\left(\sup _{t \in[0, T]}\{Z(t)\}<0\right)=\Theta(T),
$$

if near the origin the corresponding spectral measure $\mu_{A}$ has a bounded away from zero and infinity density, and for some $\delta>0$ the integral $\int_{\mathbb{R}}|\lambda|^{\delta} \mu_{A}(d \lambda)$ is finite. Our proof of Lemma 3.1 shows that any centered, stationary, separable Gaussian process $Z(\cdot)$ with absolutely integrable $A(0, \cdot)$, has at least exponentially decaying persistence probabilities (so neither bounded away from zero density near the origin nor having $\int_{\mathbb{R}}|\lambda|^{\delta} \mu_{A}(d \lambda)<\infty$, are required for such exponential decay). It further raises the natural question what is the precise necessary and sufficient condition for having at least exponential decay of persistence probabilities of such processes.

As an application of Theorem 1.2, we sharpen some of the results of Sak about asymptotic persistence probabilities for a certain family of $\nabla \phi$-interface models. Specifically, consider the $\mathbb{R}_{+} \times \mathbb{Z}^{d}$-indexed centered Gaussian process $\left\{\phi_{t}(\mathbf{x})\right\}$ given by the unique strong solution of the corresponding (Langevin) system of interacting diffusion processes:

$$
d \phi_{t}(\mathbf{x})=\left\{-\phi_{t}(\mathbf{x})+\sum_{\mathbf{y} \neq \mathbf{x}} q(\mathbf{y}-\mathbf{x}) \phi_{t}(\mathbf{y})\right\} d t+\sqrt{2} d B_{t}(\mathbf{x}), \quad \phi_{0}(\mathbf{x})=0 .
$$

Here $\left\{B_{t}(\mathbf{x})\right\}_{\mathbf{x} \in \mathbb{Z}^{d}}$ is a collection of independent standard Brownian motions, and we make the following assumptions about $q: \mathbb{Z}^{d} \mapsto \mathbb{R}_{+}$.

Assumption 1.7. The function $q: \mathbb{Z}^{d} \mapsto \mathbb{R}_{+}$satisfies the following four conditions:

(a) $q(\mathbf{x})=q(-\mathbf{x})$,

(b) There exists $R<\infty$ such that $q(\mathbf{x})=0$ whenever $\|\mathbf{x}\|_{2} \geq R$,

(c) $\sum_{\mathbf{x} \neq \mathbf{0}} q(\mathbf{x})=1$

(d) The additive group generated by $\left\{\mathbf{x} \in \mathbb{Z}^{d}: q(\mathbf{x})>0\right\}$ is $\mathbb{Z}^{d}$.

Such $\nabla \phi$ and other, closely related, models received much interest in mathematical physics and probability literature (c.f. [Deu, FS, Gar, GOS, Ham] and the references therein). It is not hard to verify that a standard approximation argument proves the existence of a unique strong solution of (1.15) (that is, a stochastic process $\phi_{t}(\mathbf{x}) \in C\left([0, \infty), \mathcal{E}^{\prime}\right)$ for $\mathcal{E}^{\prime}=\left\{\mathbf{x}: \sum_{i}(1+\|i\|)^{-2 p}|x(i)|^{2}<\infty\right.$, for some $p \geq 1\}$, adapted to the filtration $\sigma\left(B_{s}(\mathbf{x}): \mathbf{x} \in \mathbb{Z}^{d}, s \leq t\right)$ and satisfying (1.15) $)$. Further, there exists a random walk representation for the space-time correlations of (1.15) (c.f. [DD, Deu2]; see also the references therein for other interacting diffusion processes admitting a random walk representation for their correlations). From this random walk representation we have that the covariance of the centered Gaussian process $g_{t}:=\phi_{t}(\mathbf{0})$ is

$$
\Gamma^{(q)}(s, t):=\int_{|s-t|}^{s+t} \mathbb{P}\left(S_{u}^{(q)}=\mathbf{0}\right) d u
$$

Here $\left\{S_{u}^{(q)}\right\}_{u \geq 0}$ denotes the continuous time random walk on $\mathbb{Z}^{d}$, starting at $S_{0}^{(q)}=\mathbf{0}$ which upon its arrival to any site $\mathbf{x} \in \mathbb{Z}^{d}$ waits for an independent, Exponential(1) time, then moves with probability $q(\mathbf{y}-\mathbf{x})$ to $\mathbf{y} \in \mathbb{Z}^{d} \backslash\{\mathbf{x}\}$. The correlation of the process $\left\{g_{t}\right\}$ is consequently of the form

$$
C_{\rho}(s, t)=\frac{I_{\rho}(s+t)-I_{\rho}(|s-t|)}{\sqrt{I_{\rho}(2 s) I_{\rho}(2 t)}},
$$


for $I_{\rho}(\cdot)$ of (1.4), where $\rho(u):=\mathbb{P}\left(S_{u}^{(q)}=\mathbf{0}\right)$ is bounded, strictly positive and regularly varying (see the proof of Corollary 1.10). More generally, replacing $\mathbb{P}\left(S_{u}^{(q)}=\mathbf{0}\right)$ by some other regularly varying function $\rho$, our next theorem provides asymptotic decay of persistence probabilities for any centered Gaussian process $\left\{Y_{\rho}(t)\right\}_{t>0}$ having correlation $C(s, t):=\mathbb{E}\left[Y_{\rho}(t) Y_{\rho}(s)\right]$ of the form (1.17) for some $\rho \in \mathcal{R}_{\gamma}$. To this end, for $\gamma>1$ we may utilize the corresponding limiting correlation function

$$
\bar{C}_{\rho}(s, t):=\lim _{k \rightarrow \infty} C_{\rho}(s+k, t+k)=1-\frac{I_{\rho}(|s-t|)}{I_{\rho}(\infty)} .
$$

For $\gamma \in[0,1)$ we shall instead consider the universal limiting correlation functions associated with the Lamperti transformation $t=e^{u}$ (see [Lam]). That is,

$$
C_{\gamma}^{\star}(v, u):=\lim _{k \rightarrow \infty} C_{\rho}\left(e^{v+k}, e^{u+k}\right)=\cosh (|u-v| / 2)^{1-\gamma}-\sinh (|u-v| / 2)^{1-\gamma} .
$$

The latter functions appear in the physics literature when studying persistence of Gaussian processes driven by linear stochastic differential equations (see [KKMCBS, MB2]).

Theorem 1.8. Suppose the process $\left\{Y_{\rho}(\cdot)\right\}$ has correlation function of the form (1.17) for some $\rho \in \mathcal{R}_{\gamma}$ and let $I_{\widetilde{\rho}}(\cdot)$ denote the primitive of $\widetilde{\rho}(s):=s \rho(s)$.

(a) If $\gamma>2$ or $\gamma=2$ and $I_{\widetilde{\rho}}(\infty)<\infty$, then

$$
-\lim _{T \rightarrow \infty} \frac{1}{T} \log \mathbb{P}\left(\sup _{t \in[1, T]}\left\{Y_{\rho}(t)\right\}<0\right)=b\left(\bar{C}_{\rho}\right) \in(0, \infty),
$$

provided $\rho(\cdot)$ is uniformly bounded away from zero on compacts.

(b) If $\gamma \in[0,1)$, then

$$
-\lim _{T \rightarrow \infty} \frac{1}{\log T} \log \mathbb{P}\left(\sup _{t \in[1, T]}\left\{Y_{\rho}(t)\right\}<0\right)=b\left(C_{\gamma}^{\star}\right) \in(0, \infty) .
$$

(c) If $\gamma \in(1,2)$ or $\gamma=2$ and $I_{\widetilde{\rho}}(\infty)=\infty$, then

$$
-\log \mathbb{P}\left(\sup _{t \in[1, T]}\left\{Y_{\rho}(t)\right\}<0\right)=\Theta\left(a_{\widetilde{\rho}}(T)\right)
$$

Remark 1.9. Note that for $\gamma \in[0,1)$ we get the same persistence power exponent $b\left(C_{\gamma}^{\star}\right)$ for all $\rho \in \mathcal{R}_{\gamma}$ (which is not the case when $\gamma>2$ ).

We have the following immediate application of Theorem 1.8 for $\rho^{(q)}(u):=\mathbb{P}\left(S_{u}^{(q)}=\mathbf{0}\right)$.

Corollary 1.10. Fixing $d \in \mathbb{N}$ and $q: \mathbb{Z}^{d} \mapsto \mathbb{R}_{+}$satisfying Assumption 1.7 , let $g_{t}=\phi_{t}(\mathbf{0})$ for $\phi_{t}(\mathbf{x})$ which is the unique strong solution of (1.15).

(a) If $d=1$ then

$$
-\frac{1}{\log T} \log \mathbb{P}\left(\sup _{t \in[1, T]}\left\{g_{t}\right\}<0\right)=b\left(C_{1 / 2}^{\star}\right) \in(0, \infty) .
$$

(b) If $d=3$ then

$$
-\log \mathbb{P}\left(\sup _{t \in[1, T]}\left\{g_{t}\right\}<0\right)=\Theta(\sqrt{T} \log T) .
$$

(c) If $d=4$ then

$$
-\log \mathbb{P}\left(\sup _{t \in[1, T]}\left\{g_{t}\right\}<0\right)=\Theta\left(\frac{T \log \log T}{\log T}\right) .
$$


(d) If $d \geq 5$ then $\rho^{(q)}(u)=\mathbb{P}\left(S_{u}^{(q)}=\mathbf{0}\right) \in \mathcal{R}_{d / 2}$ and

$$
-\lim _{T \rightarrow \infty} \frac{1}{T} \log \mathbb{P}\left(\sup _{t \in[1, T]}\left\{g_{t}\right\}<0\right)=b\left(\bar{C}_{\rho^{(q)}}\right) \in(0, \infty) .
$$

(e) For jump rates $q_{d}: \mathbb{Z}^{d} \mapsto \mathbb{R}_{+}$satisfying Assumption 1.7 and any $k \geq 0$, let $G_{k}^{\left(q_{d}\right)}$ denote the expected occupation time of $\mathbf{0}$ during $\{k, k+1, \ldots\}$ by a discrete time random walk of transition probabilities $q_{d}(\mathbf{y}-\mathbf{x})$ that starts at $\{\mathbf{0}\}$. Suppose the Green functions $G_{0}^{\left(q_{d}\right)} \rightarrow 1$ as $d \rightarrow \infty$ and $k^{2} G_{k}^{\left(q_{d}\right)}$ is uniformly bounded over $k \geq 1$ and $d \geq d_{0}$. Then,

$$
\lim _{d \rightarrow \infty} b\left(\bar{C}_{\rho^{\left(q_{d}\right)}}\right)=1 \text {. }
$$

Proof. In view of Assumption 1.7, the convergence $u^{d / 2} \mathbb{P}\left(S_{u}^{(q)}=0\right) \rightarrow c_{q}$ for some finite constant $c_{q}>0$ readily follows from the local CLT for $\left\{S_{u}^{(q)}\right\}$, as in [LL, Theorem 2.1.3]. Hence, comparing (1.16) with (1.17), parts (a)-(d) of the corollary are an immediate application of Theorem 1.8 for $\rho^{(q)}(u) \in \mathcal{R}_{d / 2}$ (indeed, part (a) of Theorem 1.8 takes care of $d \geq 5$, part (b) handles $d=1$, while part (c) deals with both $d=3$ for which $I_{\widetilde{\rho}}(T)=\Theta(\sqrt{T})$ and $d=4$ for which $\left.I_{\widetilde{\rho}}(T)=\Theta(\log T)\right)$. Part (e) is an application [DM, Theorem 1.6], the details of which are provided in Section 3 ,

Remark 1.11. Corollary 1.10 gives the exact order of decay for any $d \neq 2$, as well as existence of a limiting persistence exponent for $d=1$ and $d \geq 5$. In doing so it improves upon the earlier results of [Sak] (where the decay rate is determined for $d=1, d \geq 5$ without the existence of a limit, and decay rate upper and lower bounds within a $\log T$ factor are given for $d=2,3,4)$. Recall that $\mathbb{P}\left(S_{u}^{(q)}=0\right)=\Theta(1 / u)$ when $d=2$, hence the process $\left\{g_{t} / \sqrt{\mathbb{E} g_{t}^{2}}\right\}$ then has auto-correlation $A(s, t)=\Theta(1 / \log |t-s|)$ for $1 \ll|s-t|=\Theta(t)$. This corresponds to $\alpha=0$, a case for which Theorem 1.2 does not apply, but Proposition 1.5 predicts that

$$
(\log T)^{2} \lesssim-\log \mathbb{P}\left(\sup _{t \in[1, T]}\left\{g_{t}\right\}<0\right) \lesssim(\log T)^{3},
$$

as indeed proved in $\mathrm{Sak}]$.

Remark 1.12. As another application of Theorem 1.2, we determine the exact rate of persistence decay for stationary fractional Brownian motion of order $H \in(1 / 2,1)$ defined by the stochastic integral

$$
Y_{H}(t):=\int_{-\infty}^{t} e^{-(t-s)} d B_{H}(s)
$$

where $B_{H}($.$) is two sided fractional Brownian motion of order H \in(1 / 2,1)$. We refer to Unt for a definition of stochastic integration with respect to fBM with Hurst index $H>\frac{1}{2}$. Using [Unt, (1.1)] we find that the stationary correlation function of $Y_{H}(\cdot)$ is

$$
\Lambda_{H}(0, \tau)=e^{-\tau}+\frac{1}{\mathbb{E}\left[Y_{H}(0)^{2}\right]} \int_{0}^{\tau} e^{-(\tau-s)} R(s) d s,
$$

where

$$
R(s):=H(2 H-1) \int_{0}^{\infty} e^{-v}(v+s)^{2 H-2} d v
$$

is asymptotically of order $H(2 H-1) s^{2 H-2}$ for $s$ large. Consequently we have that

$$
\limsup _{\tau \rightarrow \infty} \frac{\Lambda_{H}(t, t+\tau)}{\tau^{2 H-2}}=\frac{H(2 H-1)}{\mathbb{E}\left[Y_{H}(0)^{2}\right]}
$$


with conditions (1.7) and (1.10) satisfied for the regularly varying $\rho(\tau)=\min \left(1, \tau^{2 H-2}\right)$. As for (1.9), recall that $\Lambda_{H}(0, \tau) \geq \Lambda_{1 / 2}(0, \tau)=e^{-\tau}$, hence by Slepian's lemma,

$$
\mathbb{E}\left[\sup _{t \in[s, s+u]}\left\{Y_{H}(t)\right\}\right] \leq \mathbb{E}\left[\sup _{t \in[s, s+u]}\left\{Y_{1 / 2}(t)\right\}\right]
$$

for the stationary ou process $Y_{1 / 2}(\cdot)$, which satisfies (1.9). Consequently, so does $Y_{H}(\cdot)$ and from Theorem 1.2 we conclude that

$$
-\log \mathbb{P}\left(\sup _{[0, T]} Y_{H}(t)<0\right)=\Theta\left(T^{2-2 H} \log T\right) .
$$

Section 2 is devoted to the proof of Theorem 1.2, with Theorem 1.2 applied in Section 3 to yield part (c) of Theorem 1.8 (and [DM, Theorem 1.6] utilized for deducing the complementary parts (a) and (b) of Theorem 1.8, as well as part (e) of Corollary 1.10).

Acknowledgment This research is the outgrowth of discussions with H. Sakagawa during a research visit of A. D. that was funded by T. Funaki from Tokyo University. We are indebted to H. Sakagawa for sharing with us a preprint of [Sak], to J. Ding for an alternative proof of Theorem 1.2(b) and to O. Zeitouni for helpful discussions. We thank G. Schehr for bringing the references [BMS, GPS] to our notice and the referees whose suggestions much improved this article.

\section{Proof of Theorem 1.2}

We first collect a few standard, well known results about Gaussian processes, that will be used throughout this paper.

2.1. Preliminaries on Gaussian processes. A key tool in our analysis is the following comparison theorem, known in literature as Slepian's lemma (see [AT, Theorem 2.2.1]).

Theorem 2.1 (Slepian's Lemma).

Suppose centered Gaussian processes $\left\{X_{t}\right\}_{t \in I}$ and $\left\{Y_{t}\right\}_{t \in I}$ are almost surely bounded on I. If

$$
\mathbb{E} X_{t}^{2}=\mathbb{E} Y_{t}^{2}, \quad \forall t \in I, \quad \mathbb{E} X_{t} X_{s} \leq \mathbb{E} Y_{t} Y_{s}, \quad \forall s, t \in I,
$$

then for any $u \in \mathbb{R}$ one has

$$
\mathbb{P}\left(\sup _{t \in I} X_{t}<u\right) \leq \mathbb{P}\left(\sup _{t \in I} Y_{t}<u\right) .
$$

Combining Slepian's lemma and sub-additivity, one has the following immediate corollary.

Corollary 2.2. If $\left\{X_{t}\right\}_{t \geq 0}$ is a centered, stationary Gaussian process of non-negative correlation function, such that $\sup _{t \in[0, T]} X_{t}$ is almost surely finite for any $T<\infty$, then the limit

$$
-\lim _{T \rightarrow \infty} \frac{1}{T} \log \mathbb{P}\left(\sup _{t \in[0, T]} X_{t}<0\right)
$$

exists in $[0, \infty]$.

The Sudakov-Fernique inequality (see [AT, Theorem 2.2.3]), is another comparison tool we use.

Theorem 2.3 (Sudakov-Fernique).

Suppose centered Gaussian processes $\left\{X_{t}\right\}_{t \in I}$ and $\left\{Y_{t}\right\}_{t \in I}$ are almost surely bounded on I. If

$$
\mathbb{E}\left(X_{t}-X_{s}\right)^{2} \leq \mathbb{E}\left(Y_{t}-Y_{s}\right)^{2}, \quad \forall s, t \in I,
$$

then one has

$$
\mathbb{E}\left[\sup _{t \in I} X_{t}\right] \leq \mathbb{E}\left[\sup _{t \in I} Y_{t}\right]
$$


We often rely on Borell-TIS inequality (see [AT, Theorem 2.1.1]) to provide concentration results for the supremum of Gaussian processes.

Theorem 2.4 (Borell-TIS). If centered Gaussian process $\left\{X_{t}\right\}_{t \in I}$ is almost surely bounded on $I$, then $\mathbb{E}\left[\sup _{t \in I} X_{t}\right]<\infty$ and for $\sigma_{I}^{2}:=\sup _{t \in I} \mathbb{E} X_{t}^{2}$ and any $u>0$,

$$
\mathbb{P}\left(\sup _{t \in I} X_{t}-\mathbb{E} \sup _{t \in I} X_{t}>u\right) \leq e^{-u^{2} / 2 \sigma_{I}^{2}} .
$$

We conclude with the standard formula for the distribution of a Gaussian process conditioned on finitely many coordinates.

Theorem 2.5. If centered Gaussian process $\left\{Z_{t}\right\}_{t>0}$ has covariance $A(\cdot, \cdot)$, then for any $\ell$ distinct indices $0 \leq t_{1}<\cdots<t_{\ell}$, conditional on $\left(Z_{t_{i}}, 1 \leq i \leq \ell\right)$ the process $Z_{t}$ has Gaussian distribution of mean

$$
m(t):=\sum_{i, j=1}^{\ell} \Omega(i, j) A\left(t, t_{i}\right) Z_{t_{j}}
$$

and covariance function

$$
\widetilde{A}(s, t):=A(s, t)-\sum_{i, j=1}^{\ell} A\left(s, t_{i}\right) \Omega(i, j) A\left(s, t_{j}\right),
$$

where $\Omega^{-1}$ is the $\ell$-dimensional covariance matrix of the centered Gaussian vector $\left(Z_{t_{i}}, 1 \leq i \leq \ell\right)$.

2.2. Proof of Theorem 1.2. We begin by showing the positivity of persistence probabilities over compact intervals for centered Gaussian processes of unit variance, non-negative correlation and a.s. continuous sample path.

Lemma 2.6. Suppose the centered Gaussian process $\{Z(t)\}$ has a.s. continuous sample paths, unit variance and non-negative correlation. Then, for any $u \in \mathbb{R}$ and compact interval $I$,

$$
\mathbb{P}\left(\sup _{t \in I}\{Z(t)\}<u\right)>0 .
$$

Proof. Let $M(I):=\sup _{t \in I}\{Z(t)\}$ and suppose that $\mathbb{P}\left(M\left(I_{1}\right)<u\right)=0$ for some $u \in \mathbb{R}$ and compact interval $I_{1}$. Representing $I_{1}$ as the disjoint union of intervals $I_{2}^{(-)}$and $I_{2}^{(+)}$each of half the length of $I_{1}$, we get by the non-negativity of correlations and Slepian's lemma (Theorem 2.1) that

$$
0=\mathbb{P}\left(M\left(I_{1}\right)<u\right) \geq \mathbb{P}\left(M\left(I_{2}^{(-)}\right)<u\right) \mathbb{P}\left(M\left(I_{2}^{(+)}\right)<u\right) .
$$

So, either $\mathbb{P}\left(M\left(I_{2}^{(-)}\right)<u\right)=0$ or $\mathbb{P}\left(M\left(I_{2}^{(+)}\right)<u\right)=0$ and proceeding inductively with the subinterval for which we have zero probability, we construct non-empty nested compact intervals $I_{k}$ of shrinking diameters such that $\mathbb{P}\left(M\left(I_{k}\right)<u\right)=0$ for all $k$. By Cantor's intersection theorem, $\bigcap_{k} I_{k}$ is a single non-random point $t_{\star}$. Thus, by the continuity of sample paths we get that a.s.

$$
\lim _{k \rightarrow \infty} M\left(I_{k}\right)=Z\left(t_{\star}\right) .
$$

Consequently,

$$
0=\lim _{k \rightarrow \infty} \mathbb{P}\left(M\left(I_{k}\right)<u\right) \geq \mathbb{P}\left(Z\left(t_{\star}\right)<u\right)>0,
$$

a contradiction which rules out our hypothesis that $\left\{M\left(I_{1}\right)<u\right\}$ has zero probability.

We recall some properties of positive, measurable slowly varying functions, that are used throughout this paper. 
Remark 2.7. For any $L \in \mathcal{R}_{0}$ (namely, positive, measurable, slowly varying function on $\mathbb{R}_{+}$), the convergence of $\frac{L(\lambda t)}{L(t)}$ to 1 is uniform over $\lambda$ in a compact subset of $(0, \infty)$ (see [BGT, Theorem 1.2.1]). Further, by the representation theorem (see [BGT, Theorem 1.3.1]), there exists then $\widetilde{L} \in \mathcal{R}_{0}$ such that

$$
\lim _{x \rightarrow \infty} \frac{\widetilde{L}(x)}{L(x)}=1
$$

and $x \mapsto x^{\eta} \widetilde{L}(x)$ is eventually increasing (decreasing) if $\eta>0(\eta<0$ resp.). That is, up to a universal constant factor (that depend on $L(\cdot)$ ), the function $x^{\eta} L(x)$ may be assumed eventually increasing (decreasing) if $\eta>0(\eta<0$ resp.).

W.l.o.g. we assume throughout that $\{Z(\cdot)\}$ has been re-scaled so that $\mathbb{E}\left[Z(t)^{2}\right]=1$ for all $t \in \mathbb{R}_{+}$ and state next three auxiliary lemmas which are needed for proving Theorem 1.2 (while deferring the proof of these lemmas to the end of the section).

Lemma 2.8. For $\alpha \geq 0$ and $\rho \in \mathcal{R}_{\alpha}$, let $L_{\rho}(x)=x^{\alpha} \rho(x) \in \mathcal{R}_{0}$ for which we further assume the eventual monotonicity properties of Remark 2.7.

(a) If $0 \leq \alpha<1$,

$$
\lim _{b \rightarrow \infty} \sup _{a \in[0, b)}\left|\frac{I_{\rho}(b)-I_{\rho}(a)}{L_{\rho}(b)\left(b^{1-\alpha}-a^{1-\alpha}\right)}-\frac{1}{1-\alpha}\right|=0 .
$$

(b) If $\alpha>1$,

$$
\lim _{b \rightarrow \infty} \sup _{a \in(b, \infty)}\left|\frac{I_{\rho}(a)-I_{\rho}(b)}{L_{\rho}(b)\left(b^{1-\alpha}-a^{1-\alpha}\right)}-\frac{1}{\alpha-1}\right|=0 .
$$

Lemma 2.9. Suppose $\rho \in \mathcal{R}_{\alpha}$ for $\alpha \geq 0$.

(a) The function $I_{\rho}(\cdot)$ is a regularly varying function of order $(1-\alpha)_{+}$and

$$
\limsup _{n, M \rightarrow \infty} \frac{M \sum_{\ell=1}^{n} \rho(\ell M)}{I_{\rho}(n M)}<\infty .
$$

If $I_{\rho}(\infty)<\infty$, then we have the stronger conclusion

$$
\limsup _{M \rightarrow \infty} M \sum_{\ell=1}^{\infty} \rho(\ell M)=0 .
$$

(b) Suppose $\alpha \in[0,1]$, with $\rho(x) \rightarrow 0$ when $\alpha=0$ and $I_{\rho}(\infty)=\infty$ when $\alpha=1$. Then, fixing $\mu>0$ we have for $M:=\mu I_{\rho}(T)$ that

$$
\lim _{T \rightarrow \infty} \sum_{\ell=1}^{\lceil T / M\rceil} \rho(\ell M)=\frac{1}{\mu} .
$$

Lemma 2.10. If the auto-correlation $A(\cdot, \cdot)$ of a centered Gaussian process $\{Z(\cdot)\}$ satisfies (1.7) for some $\rho \in \mathcal{R}_{\alpha}$ and $\alpha \in(0,1]$. Then, there exist $\eta, \delta>0$ such that

$$
\lim _{M \rightarrow \infty} \frac{1}{\log M} \sup _{s \geq M / \eta} \log \mathbb{P}\left(\sup _{t \in[s, s+M]}\{Z(t)\}<\sqrt{\delta \log M}\right)=-\infty .
$$


Proof of Theorem 1.2. Throughout the proof, all constants implied by the notation $\lesssim$ depend only on the function $A(\cdot, \cdot)$.

(a). By (1.7) there exist $\widetilde{\eta}>0$ small, $T_{\star}$ finite and $r=1-\widetilde{\eta} \in(0,1)$ such that

$$
\sup _{t \in[r T, T-\tau]} A(t, t+\tau) \lesssim \rho(\tau), \quad \forall \tau \geq 0, \quad T \geq T_{\star} .
$$

For some large universal constant $\lambda<\infty$ to be chosen in the sequel, we set $M=M(T):=\lambda I_{\rho}(T)$ and $n=n(T):=\left\lfloor\frac{(1-r) T}{2 M}\right\rfloor$, both of which diverge with $T \rightarrow \infty$ due to our assumptions on $\rho(\cdot)$. We then consider the following subset of $[r T, T]$,

$$
\mathcal{J}:=\bigcup_{\ell=1}^{n} \mathcal{J}_{2 \ell}
$$

That is, $\mathcal{J}$ is the union of every other sub-interval $\mathcal{J}_{\ell}:=\left[s_{\ell}, s_{\ell+1}\right]$, where $s_{\ell}:=r T+(\ell-1) M$ for $\ell \geq 1$, and $n(T)$ is the largest $\ell \in \mathbb{N}$ such that $s_{2 \ell+1} \leq T$. With $\mathcal{J} \subset[0, T]$, we trivially have that

$$
\mathbb{P}\left(\sup _{t \in[0, T]}\{Z(t)\}<0\right) \leq \mathbb{P}\left(\sup _{t \in \mathcal{J}}\{Z(t)\}<0\right) .
$$

For $t \in \mathcal{J}$ let $J(t)=\ell$ when $t \in \mathcal{J}_{2 \ell}$ for some $\ell \in\{1,2, \ldots, n\}$ noting that for all $s, t \in \mathcal{J}$,

$$
A(s, t) \leq \frac{1}{2} A(s, t) 1_{\{J(s)=J(t)\}}+\frac{1}{2} B(J(s), J(t)),
$$

where

$$
B(i, j):=2 \sup \left\{A(s, t): s \in \mathcal{J}_{2 i}, t \in \mathcal{J}_{2 j}\right\}, \text { if } i \neq j, \quad B(i, i)=1 .
$$

If $s, t \in \mathcal{J}$ with $J(s) \neq J(t)$, then clearly

$$
M|J(s)-J(t)| \leq|s-t| \leq 3 M|J(s)-J(t)| .
$$

Since $M=M(T) \rightarrow \infty$, we have from (2.7), (2.10) and (2.11), that with $\rho(\cdot)$ regularly varying,

$$
B(i, j) \lesssim \sup _{x \in[M|i-j|, 3 M|i-j|]} \rho(x) \lesssim \rho(M|i-j|),
$$

uniformly in $i \neq j$ and for all $M$ large enough. We thus deduce by (2.12) and (2.3) that

$$
\xi(T):=\sup _{1 \leq j \leq n}\left\{\sum_{i=1, i \neq j}^{n} B(i, j)\right\} \lesssim \sum_{\ell=1}^{n} \rho(\ell M) \lesssim \frac{I_{\rho}(n M)}{M} \leq \frac{1}{\lambda}
$$

where the right-most inequality results from having chosen $M=\lambda I_{\rho}(T)$ and $n M \leq T$ (so $I_{\rho}(n M) \leq$ $\left.I_{\rho}(T)\right)$. The universal constant on the RHS of (2.13) is independent of $\lambda$, hence there exist $\lambda=\lambda_{1}$ and $T_{\star \star}$ finite, such that $\xi(T) \leq 1 / 2$ for all $T \geq T_{\star \star}$. Using hereafter $\lambda=\lambda_{1}$ for the remainder of part (a) and the fact that $B(i, i)=1$, it follows by the Gershgorin circle theorem and the interlacing property of eigenvalues, that for any $T \geq T_{\star \star}$, the principal sub-matrices of the symmetric $n$ dimensional matrix $\mathbf{B}=\{B(i, j)\}$ have all their eigenvalues within $[1 / 2,3 / 2]$. In particular, $\mathbf{B}$ is positive definite, and with $\left\{X_{\ell}\right\}_{\ell=1}^{n}$ denoting the centered Gaussian random vector of covariance matrix $\mathbf{B}$, upon applying the argument in [DM, display following (2.5)] for principal sub-matrices of $\mathbf{B}$, we get that for any $L>0$ and all $1 \leq i_{1}<\cdots<i_{k} \leq n$,

$$
\mathbb{P}\left(\sup _{l=1}^{k}\left\{X_{i_{l}}\right\}<-\sqrt{L}\right) \leq 3^{k / 2} \mathbb{P}\left(X_{1}>\sqrt{2 L / 3}\right)^{k} .
$$

Next, we denote by $\{\bar{Z}(t), t \in \mathcal{J}\}$ the centered Gaussian process which has the same law as $\{Z(t)\}$ when restricted to each sub-interval $\mathcal{J}_{2 \ell}$, while being independent across different sub-intervals and 
independent of the random vector $\left\{X_{\ell}\right\}_{\ell=1}^{n}$. Then by (2.9), upon applying Slepian's lemma we get that for all $L>0$ and $T \geq \max \left(T_{\star}, T_{\star \star}\right)$,

$$
\mathbb{P}\left(\sup _{t \in \mathcal{J}}\{Z(t)\}<0\right) \leq \mathbb{E}\left[\prod_{\ell=1}^{n(T)}\left(\mathbb{P}\left(\sup _{t \in \mathcal{J}_{2 \ell}}\{\bar{Z}(t)\}<\sqrt{L}\right)+1_{\left\{X_{\ell}<-\sqrt{L}\right\}}\right)\right]
$$

(c.f. [DM, (2.4)] for a more detailed version of this argument). Utilizing now (2.14), we deduce from (2.15) in a similar manner as the derivation of [DM, (2.6)] that for all $L>0$,

$$
\begin{aligned}
\mathbb{P}\left(\sup _{t \in \mathcal{J}}\{Z(t)\}<0\right) & \leq\left[f(\delta, T)+\sqrt{3} \mathbb{P}\left(X_{1}>\sqrt{2 L / 3}\right)\right]^{n(T)} \\
& \leq 2^{n(T)} \max \left[f(\delta, T), \sqrt{3} \mathbb{P}\left(X_{1}>\sqrt{2 L / 3}\right)\right]^{n(T)},
\end{aligned}
$$

where

$$
f(\delta, T):=\sup _{\ell=1}^{n(T)} \mathbb{P}\left(\sup _{t \in \mathcal{J}_{2 \ell}}\{Z(t)\}<\sqrt{L}\right) .
$$

Moreover, setting $L=L(T):=\delta \log I_{\rho}(T)$, upon considering (2.6) for the intervals $\mathcal{J}_{2 \ell}$ within $[r T, T]$, of length $M(T)=\lambda_{1} I_{\rho}(T)$ each, we can choose $\delta>0$ small enough so that

$$
\lim _{T \rightarrow \infty} \frac{\log f(\delta, T)}{\log I_{\rho}(T)}=-\infty
$$

Since $n(T) L(T)=\frac{(1-r) \delta}{2 \lambda_{1}} a_{\rho}(T)$, considering $-a_{\rho}(T)^{-1} \log$ of both sides of (2.16) , we deduce from the usual tail estimates for the $N(0,1)$ law of $X_{1}$, that

$$
-\limsup _{T \rightarrow \infty} \frac{1}{a_{\rho}(T)} \log \mathbb{P}\left(\sup _{t \in \mathcal{J}}\{Z(t)\}<0\right) \geq \frac{(1-r) \delta}{6 \lambda_{1}}>0
$$

(with a negligible contribution of $f(\delta, T)$ due to (2.17) $)$. Combined with (2.8) this yields the stated upper bound (1.8).

(b) Step I. We first show that suffices for (1.11) to have for some $r \in(0,1)$ and finite $C_{1}, T_{1}$,

$$
\mathbb{P}\left(\sup _{t \in[r T, T]}\{Z(t)\}<0\right) \geq e^{-C_{1} a_{\rho}(T)} \quad \forall T \geq T_{1} .
$$

To this effect, for $T \geq T_{1}$ set $m=m(T):=\left\lceil\frac{\log \left(T / T_{1}\right)}{\log (1 / r)}\right\rceil$ and

$$
\widehat{a}_{\rho}(T):=\sum_{i=1}^{m} a_{\rho}\left(T_{i}\right), \quad T_{i}:=r^{1-i} T_{1}, \quad i \geq 0,
$$

so that $T_{m} \in[T, T / r]$. With $A(\cdot, \cdot)$ non-negative, by Slepian's lemma and (2.18) we have

$$
\begin{aligned}
\mathbb{P}\left(\sup _{t \in[0, T]}\{Z(t)\}<0\right) & \geq \mathbb{P}\left(\sup _{t \in\left[0, T_{0}\right]}\{Z(t)\}<0\right) \prod_{i=1}^{m} \mathbb{P}\left(\sup _{t \in\left[T_{i-1}, T_{i}\right]}\{Z(t)\}<0\right) \\
& \geq \mathbb{P}\left(\sup _{t \in\left[0, T_{0}\right]}\{Z(t)\}<0\right) e^{-C_{1} \widehat{a}_{\rho}(T)} .
\end{aligned}
$$

Next, by Lemma 2.6 the event $\left\{\sup _{t \in\left[0, T_{0}\right]}\{Z(t)\}<0\right\}$ has positive probability, so considering the limsup as $T \rightarrow \infty$ of $-a_{\rho}(T)^{-1} \log$ of both sides of the preceding inequality, we get (1.11) upon showing that

$$
\widehat{a}_{\rho}(T) \lesssim a_{\rho}(T)
$$


To this end, recall by Lemma 2.9 that $t \mapsto I_{\rho}(t)$ is regularly varying of order $1-\alpha$, hence $a_{\rho}(t)=$ $t \log I_{\rho}(t) / I_{\rho}(t)$ is regularly varying of order $\alpha>0$. Thus, there exists $K \geq 1$ finite such that $a_{\rho}\left(T_{i-1}\right) \leq r^{\alpha / 2} a_{\rho}\left(T_{i}\right)$ for all $i \geq K$, from which we deduce that

$$
\widehat{a}_{\rho}(T) \leq \sum_{i=1}^{K} a_{\rho}\left(T_{i}\right)+a_{\rho}\left(T_{m}\right) \sum_{l=0}^{\infty} r^{\alpha l / 2} .
$$

The first sum on the RHS is finite and independent of $T$. Further, with $\alpha>0$ and $r \in(0,1)$, the same applies for the second sum there. Lastly, since $a_{\rho}(\cdot)$ is regularly varying of positive order, $a_{\rho}\left(T_{m}\right) \lesssim a_{\rho}(T)$, yielding (2.21) and thereby (1.11).

Step II. We proceed to verify (2.18) for $r=1-\eta \in(0,1)$ and $\eta$ small enough so that by (1.10) in addition to (2.7), we further have for all $T$ large enough

$$
\rho(\tau) \lesssim \inf _{t \in[r T, T-\tau]} A(t, t+\tau), \quad \forall \tau \geq 0
$$

Then, for such $T$ large, set $M=M(T)=\lambda I_{\rho}(T)$ for some finite $\lambda$ to be chosen in the sequel, and cover the interval $[r T, T]$ by

$$
\mathcal{J}:=\bigcup_{\ell=1}^{3 n^{\prime}} \overline{\mathcal{J}_{\ell}}
$$

for the corresponding open sub-intervals $\mathcal{J}_{\ell}=\left(s_{\ell}, s_{\ell+1}\right)$, with $s_{\ell}=r T+(\ell-1) M$ for $\ell=1, \ldots, n^{\prime}$ and $n^{\prime}=n^{\prime}(T):=\left\lceil\frac{(1-r) T}{3 M}\right\rceil$ the smallest integer for which $[r T, T] \subseteq \mathcal{J}$. An application of Slepian's lemma gives

$$
\mathbb{P}\left(\sup _{t \in[r T, T]}\{Z(t)\}<0\right) \geq \prod_{i=0}^{2} \mathbb{P}\left(\sup _{t \in \mathcal{J}^{i}}\{Z(t)<0), \quad \mathcal{J}^{i}:=\bigcup_{\ell=1}^{n^{\prime}} \overline{\mathcal{J}}_{3 \ell-i}\right.
$$

We will show that for $i=0$,

$$
\liminf _{T \rightarrow \infty} \frac{1}{a_{\rho}(T)} \log \mathbb{P}\left(\sup _{t \in \mathcal{J}^{i}}\{Z(t)\}<0\right)>-\infty
$$

and with the same reasoning applicable for $i=1,2$, the bound (2.18) follows for suitably chosen $C_{1}, T_{1}$ finite. Turning to show (2.23), we take $L=L(T):=\beta \log I_{\rho}(T)$ for some $\beta=\beta(\lambda)$ finite to be determined later, and get a lower bound by enforcing the event

$$
\Gamma:=\left\{-\sqrt{L}<Z\left(s_{3 \ell-1}\right)<-(3 / 4) \sqrt{L}, \quad \ell=1, \ldots, n^{\prime}\right\},
$$

which is measurable with respect to the $\sigma$-algebra $\mathcal{F}:=\sigma\left(Z\left(s_{3 \ell-1}\right), 1 \leq \ell \leq n^{\prime}\right)$. Indeed,

$$
\mathbb{P}\left(\sup _{\ell=1}^{n^{\prime}} \sup _{t \in \overline{\mathcal{J}}_{3 \ell}}\{Z(t)\}<0\right) \geq \mathbb{E}\left[\mathbb{P}\left(\bigcap_{\ell=1}^{n^{\prime}} \sup _{t \in \overline{\mathcal{J}}_{3 \ell}}\{Z(t)\}<0 \mid \mathcal{F}\right) 1_{\Gamma}\right]
$$

and proceeding to bound the RHS of (2.24), let $m(t)$ denote the conditional mean of $Z(t)$ given $\mathcal{F}$. We claim that for some choice of $\lambda=\lambda_{2}$ and $C_{2}=C_{2}(\lambda)>0$ one has that

$$
\begin{aligned}
\Gamma \quad \Longrightarrow \quad \sqrt{C_{2} L} & \geq \sup _{t \in \mathcal{J}^{0}}\{m(t)\}, \\
\operatorname{Cov}(Z(u), Z(v) \mid \mathcal{F}) & \geq 0 .
\end{aligned}
$$


We next complete the proof of (2.23) assuming both (2.25) and (2.26) hold (deferring to Step III the proof of the latter estimates). Indeed, by Slepian's lemma it then follows that

$$
\begin{aligned}
\mathbb{P}\left(\bigcap_{\ell=1}^{n^{\prime}} \sup _{\left.t \in \overline{\mathcal{J}}_{3 \ell}\{Z(t)\}<0 \mid \mathcal{F}\right) 1_{\Gamma}} \geq \prod_{\ell=1}^{n^{\prime}} \mathbb{P}\left(\sup _{t \in \overline{\mathcal{J}}_{3 \ell}}\{Z(t)\}<0 \mid \mathcal{F}\right) 1_{\Gamma}\right. \\
\geq \prod_{\ell=1}^{n^{\prime}} \mathbb{P}\left(\sup _{t \in \overline{\mathcal{J}}_{3 \ell}}\{Z(t)-m(t)\}<\sqrt{C_{2} L} \mid \mathcal{F}\right) 1_{\Gamma} .
\end{aligned}
$$

Conditional on $\mathcal{F}$, the centered normal random variable $Y_{u, v}:=Z(u)-m(u)-Z(v)+m(v)$ has variance $\mathbb{E}\left[Y_{u, v}^{2} \mid \mathcal{F}\right] \leq \mathbb{E}\left[(Z(u)-Z(v))^{2}\right]$. Thus, by the Sudakov-Fernique inequality (Theorem [2.3), for any $s, u>0$, a.s.

$$
\mathbb{E}\left[\sup _{t \in[s, s+u]}\{Z(t)-m(t)\} \mid \mathcal{F}\right] \leq \mathbb{E}\left[\sup _{t \in[s, s+u]}\{Z(t)\}\right]
$$

Thus, from (1.9) there exist $u_{0}>0$ and $K<\infty$ (independent of $T$ and $M$ ), such that a.s.

$$
\sup _{s \geq 0} \mathbb{E}\left[\sup _{t \in\left[s, s+u_{0}\right]}\{Z(t)-m(t)\} \mid \mathcal{F}\right] \leq K
$$

Upon covering $\mathcal{J}_{3 \ell}$ by intervals of length $u_{0}$, in each of which we apply the Borell-TIS inequality (Theorem 2.4), for the conditional Gaussian centered process $\{Z(t)-m(t)\}$ of maximal variance one, we get by a union bound that a.s.

$$
\begin{aligned}
\mathbb{P}\left(\sup _{t \in \mathcal{J}_{3 \ell}}\{Z(t)-m(t)\} \geq \sqrt{C_{2} L} \mid \mathcal{F}\right) & \leq\left\lceil M / u_{0}\right\rceil \sup _{s \geq 0} \mathbb{P}\left(\sup _{t \in\left[s, s+u_{0}\right]}\{Z(t)-m(t)\} \geq \sqrt{C_{2} L} \mid \mathcal{F}\right) \\
& \leq \frac{2 M}{u_{0}} \exp \left\{-\frac{1}{2}\left(\sqrt{C_{2} L}-K\right)^{2}\right\} .
\end{aligned}
$$

With $M=\lambda_{2} I_{\rho}(T), L=\beta \log I_{\rho}(T)$ and $I_{\rho}(T) \uparrow \infty$, upon taking $\beta>2 / C_{2}$ the RHS is bounded by $1 / 2$ for all $T$ large enough. In this case, we deduce from (2.24) and (2.27) that

$$
\begin{aligned}
\mathbb{P}\left(\sup _{t \in \mathcal{J}^{0}}\{Z(t)\}<0\right) \geq 2^{-n^{\prime}} \mathbb{P}(\Gamma) & =2^{-n^{\prime}} \mathbb{P}\left(\bigcap_{\ell=1}^{n^{\prime}}\left\{\frac{3}{4} \sqrt{L}<Z\left(s_{3 \ell-1}\right)<\sqrt{L}\right\}\right) \\
& \geq 12^{-n^{\prime} / 2} \mathbb{P}\left(\frac{3}{4} \sqrt{2 L}<Z<\sqrt{2 L}\right)^{n^{\prime}},
\end{aligned}
$$

where noting that by (2.28) all eigenvalues of the covariance matrix $\mathbf{I}+\boldsymbol{\Delta}$ are within $[1 / 2,3 / 2]$, the last inequality follows by the same argument employed in DM, display following (2.5)]. Considering the limit as $T \rightarrow \infty$ of $-a_{\rho}(T)^{-1} \log$ of both sides, results with

$$
\liminf _{T \rightarrow \infty} \frac{1}{a_{\rho}(T)} \log \mathbb{P}\left(\sup _{t \in \mathcal{J}^{0}}\{Z(t)\}<0\right) \geq-\frac{(1-r) \beta}{16 \lambda_{2}},
$$

thereby establishing (2.23), and consequently (2.18).

Step III. It remains only to establish (2.25) and (2.26). To this end, setting $\boldsymbol{\Delta}$ the $n^{\prime}$-dimensional matrix of non-negative entries

$$
\Delta\left(\ell, \ell^{\prime}\right):=\operatorname{Cov}\left(Z\left(s_{3 \ell-1}\right), Z\left(s_{3 \ell^{\prime}-1}\right)\right) \text { for } 1 \leq \ell \neq \ell^{\prime} \leq n^{\prime}, \quad \Delta(\ell, \ell):=0
$$


we claim that there exists $\lambda_{2}$ such that for all $\lambda \geq \lambda_{2}$ the following estimates hold simultaneously:

$$
\begin{array}{r}
\max \left\{\max _{\ell=1}^{n^{\prime}} \sum_{\ell^{\prime}=1}^{n^{\prime}} \Delta\left(\ell, \ell^{\prime}\right), \sup _{t \in \mathcal{J}^{0}} \sum_{\ell=1}^{n^{\prime}} A\left(t, s_{3 \ell-1}\right)\right\} \leq \frac{1}{2}, \\
\max _{a, b=1, a \neq b}^{n^{\prime}} \sup _{v \in \overline{\mathcal{J}}_{3 a}, u \in \overline{\mathcal{J}}_{3 b}} \frac{1}{A(u, v)} \sum_{\ell=1}^{n^{\prime}} A\left(u, s_{3 \ell-1}\right) A\left(v, s_{3 \ell-1}\right) \leq \frac{1}{2}, \\
\max _{a, b=1}^{n^{\prime}} \sup _{v \in \overline{\mathcal{J}}_{3 a}} \frac{1}{A\left(v, s_{3 b-1}\right)} \sum_{\ell=1}^{n^{\prime}} \Delta(b, \ell) A\left(v, s_{3 \ell-1}\right) \leq \frac{1}{2} .
\end{array}
$$

While deferring the proof of (2.28)-(2.30) to Step IV, we fix hereafter $\lambda=\lambda_{2}$ and rely on these bounds to establish (2.23). Indeed, setting the vectors

$$
\mathbf{y}(t):=\left[A\left(t, s_{3 \ell-1}\right), 1 \leq \ell \leq n^{\prime}\right], \quad \mathbf{z}:=\left[Z\left(s_{3 \ell-1}\right), 1 \leq \ell \leq n^{\prime}\right]
$$

and utilizing Theorem 2.5, the conditional mean of $Z(t)$ given $\mathcal{F}$ is

$$
m(t)=\left\langle\mathbf{y}(t),(\mathbf{I}+\boldsymbol{\Delta})^{-1} \mathbf{z}\right\rangle=\sum_{k=0}^{\infty}\left\langle\mathbf{y}(t), \Delta^{2 k}(\mathbf{I}-\boldsymbol{\Delta}) \mathbf{z}\right\rangle
$$

where the expansion as a power series requires that the operator norm of $\boldsymbol{\Delta}$ is less than 1 . To verify this, recall that the operator norm of a symmetric matrix is bounded by the maximum row sum, which coupled with (2.28) gives $\|\boldsymbol{\Delta} \mathbf{1}\|_{\infty} \leq 1 / 2$. In view of the latter bound on $\boldsymbol{\Delta}$, the event $\Gamma$ implies that

$$
\left\langle\mathbf{y}(t), \boldsymbol{\Delta}^{2 k}(\mathbf{I}-\boldsymbol{\Delta}) \mathbf{z}\right\rangle \leq-\sqrt{L}\left\langle\mathbf{y}(t), \boldsymbol{\Delta}^{2 k}\left[\frac{3}{4} \mathbf{1}-\boldsymbol{\Delta} \mathbf{1}\right]\right\rangle \leq-\frac{\sqrt{L}}{4}\left\langle\mathbf{y}(t), \boldsymbol{\Delta}^{2 k} \mathbf{1}\right\rangle .
$$

In particular, this is negative for any $k \geq 1$, hence under $\Gamma$,

$$
m(t) \leq\langle\mathbf{y}(t),(\mathbf{I}-\boldsymbol{\Delta}) \mathbf{z}\rangle \leq-\frac{\sqrt{L}}{4}\langle\mathbf{y}(t), \mathbf{1}\rangle=-\frac{\sqrt{L}}{4} \sum_{\ell=1}^{n^{\prime}} A\left(t, s_{3 \ell-1}\right) .
$$

Further, recall that for any $t \in \mathcal{J}^{0}$ the elements of $\left\{\left|t-s_{3 \ell-1}\right| / M\right\}$ are of the form $\{\theta+3 \mathbb{Z}\}$, for some $\theta=\theta(t) \in[1,2]$. Hence, with $\rho(\cdot)$ and $I_{\rho}(\cdot)$ regularly varying, by (2.22) and (2.5) (for $\left.3 M / I_{\rho}((1-r) T) \rightarrow \mu=c \lambda_{2}\right)$

$$
\inf _{t \in \mathcal{J}^{0}} \sum_{\ell=1}^{n^{\prime}} A\left(t, s_{3 l-1}\right) \gtrsim \inf _{\theta \in[1,2]}\left\{\sum_{k=0}^{n^{\prime}-1} \rho((\theta+3 k) M)\right\} \gtrsim \frac{1}{\lambda_{2}} .
$$

Combining (2.31) and (2.32), we get the existence of $C_{2}$ independent of $T$ and $L$ for which(2.25) holds. Proceeding to bound the covariance of the conditional process across blocks, we set

$$
a_{k}(u, v):=\sum_{\ell, \ell^{\prime}=1}^{n^{\prime}} A\left(u, s_{3 \ell-1}\right) \Delta^{k}\left(\ell, \ell^{\prime}\right) A\left(v, s_{3 \ell^{\prime}-1}\right)
$$

for $u \in \mathcal{J}_{3 j}, v \in \mathcal{J}_{3 j^{\prime}}$ with $1 \leq j \neq j^{\prime} \leq n^{\prime}$, and use Theorem 2.5 to note that

$$
\operatorname{Cov}(Z(u), Z(v) \mid \mathcal{F})=A(u, v)-\sum_{k=0}^{\infty}(-1)^{k} a_{k}(u, v) \geq A(u, v)-\sum_{k=0}^{\infty} a_{2 k}(u, v),
$$


where $\boldsymbol{\Delta}^{0}:=\mathbf{I}$. Note that by (2.30), for any $k \geq 1$,

$$
\begin{aligned}
a_{k}(u, v) & =\sum_{\ell, \ell^{\prime \prime}=1}^{n^{\prime}} A\left(u, s_{3 \ell-1}\right) \Delta^{k-1}\left(\ell, \ell^{\prime \prime}\right) \sum_{\ell^{\prime}=1}^{n^{\prime}} \Delta\left(\ell^{\prime \prime}, \ell^{\prime}\right) A\left(v, s_{3 \ell^{\prime}-1}\right) \\
& \leq \frac{1}{2} \sum_{\ell, \ell^{\prime}=1}^{n^{\prime}} A\left(u, s_{3 \ell-1}\right) \Delta^{k-1}\left(\ell, \ell^{\prime \prime}\right) A\left(v, s_{3 \ell^{\prime \prime}-1}\right)=\frac{1}{2} a_{k-1}(u, v)
\end{aligned}
$$

and consequently, for any $k \geq 0$, by (2.29),

$$
a_{k}(u, v) \leq\left(\frac{1}{2}\right)^{k} a_{0}(u, v) \leq\left(\frac{1}{2}\right)^{k+1} A(u, v) .
$$

Combining (2.33) and (2.34) we deduce that

$$
\operatorname{Cov}(Z(u), Z(v) \mid \mathcal{F}) \geq A(u, v)\left[1-\sum_{k=0}^{\infty}\left(\frac{1}{2}\right)^{2 k+1}\right] \geq 0 .
$$

thus verifying (2.26) as well.

Step IV. In proving (2.28)-(2.30) we repeatedly use properties of regularly varying functions, and in particular, having $\alpha>0$, assume hereafter WLOG that $\rho(\cdot)$ is eventually non-increasing (see Remark 2.7). Starting with (2.28), note that by the same argument used for deriving (2.32),

$$
\sup _{t \in \mathcal{J}^{0}}\left\{\sum_{\ell=1}^{n^{\prime}} A\left(t, s_{3 \ell-1}\right)\right\} \lesssim \sup _{\theta \in[1,2]}\left\{\sum_{k=0}^{n^{\prime}-1} \rho((\theta+3 k) M)\right\} \lesssim \frac{1}{\lambda} .
$$

The same calculation shows that

$$
\max _{\ell=1}^{n^{\prime}} \sum_{\ell^{\prime}=1}^{n^{\prime}} \Delta\left(\ell, \ell^{\prime}\right) \lesssim \sum_{k=1}^{n^{\prime}} \rho(3 k M) \lesssim \frac{1}{\lambda},
$$

so choosing $\lambda$ large enough guarantees that (2.28) holds. Next, in view of (2.7), (2.22) and having $\rho(\cdot)$ regularly varying and eventually non-increasing, the LHS of (2.29) and (2.30) are both bounded up to a universal constant multiplicative factor, by

$$
\rho(M)+\max _{1 \leq a \leq b \leq n^{\prime}}\left\{R_{\left[1, n^{\prime}\right]}\right\},
$$

where setting $I_{1}=[1, a-1], I_{2}=[a+1,(a+b) / 2], I_{3}=[(a+b) / 2, b-1], I_{4}=\left[b+1, n^{\prime}\right]$,

$$
R_{\left[1, n^{\prime}\right]}:=\sum_{\ell \neq a, b}^{n^{\prime}} \frac{\rho\left(\left|s_{\ell}-s_{a}\right|\right) \rho\left(\left|s_{\ell}-s_{b}\right|\right)}{\rho\left(\left|s_{a}-s_{b}\right|\right)}=\sum_{i=1}^{4} R_{I_{i}},
$$

and $R_{I_{i}}$ corresponds to the sum over $\ell \in I_{i}$. It thus suffices to show that $\max _{i, a, b} R_{I_{i}} \lesssim 1 / \lambda$ (so choosing $\lambda$ large enough guarantees that also (2.29) and (2.30) hold). Now with $\rho(\cdot)$ eventually non-increasing, we have that for $M$ large enough and all $a \leq b$,

$$
R_{I_{1}} \leq \sum_{\ell=1}^{a-1} \rho\left(s_{a}-s_{\ell}\right), \quad R_{I_{4}} \leq \sum_{\ell=b+1}^{n^{\prime}} \rho\left(s_{\ell}-s_{b}\right) .
$$


Further, $I_{2}$ and $I_{3}$ are empty unless $a<b$, in which case $s_{a}^{b}:=s_{b}-s_{a} \geq M$ and

$$
R_{I_{2}}=\sum_{\ell=a+1}^{(a+b) / 2} \frac{\rho\left(s_{b}-s_{\ell}\right)}{\rho\left(s_{a}^{b}\right)} \rho\left(s_{\ell}-s_{a}\right) \leq \sup _{\theta \in[1 / 2,1]}\left\{\frac{\rho\left(\theta s_{a}^{b}\right)}{\rho\left(s_{a}^{b}\right)}\right\} \sum_{\ell=a+1}^{(a+b) / 2} \rho\left(s_{\ell}-s_{a}\right) \leq C \sum_{\ell=a+1}^{(a+b) / 2} \rho\left(s_{\ell}-s_{a}\right),
$$

while by the same reasoning also

$$
R_{I_{3}}=\sum_{\ell>(a+b) / 2}^{b-1} \frac{\rho\left(s_{\ell}-s_{a}\right)}{\rho\left(s_{a}^{b}\right)} \rho\left(s_{b}-s_{\ell}\right) \leq C \sum_{\ell>(a+b) / 2}^{b-1} \rho\left(s_{b}-s_{\ell}\right) .
$$

Combining the latter four bounds, we conclude that

$$
\max _{i, a, b}\left\{R_{I_{i}}\right\} \leq C \sum_{\ell=1}^{n^{\prime}} \rho(\ell M) \lesssim \frac{1}{\lambda}
$$

as claimed.

Proof of Proposition 1.5. The bulk of the proof of Theorem 1.2 dealt with $\sup _{t \in[r T, T]}\{Z(t)\}$ for some fixed $r \in(0,1)$. This part of the proof applies even for $\rho \in \mathcal{R}_{0}$, under our extra assumptions that $\rho(\cdot)$ is eventually non-increasing and decays to 0 at $\infty$. Thus, for some $r \in(0,1)$

$$
\begin{aligned}
-\infty< & \liminf _{T \rightarrow \infty} \frac{1}{a_{\rho}(T)} \log \mathbb{P}\left(\sup _{t \in[r T, T]}\{Z(t)\}<0\right) \\
& \leq \limsup _{T \rightarrow \infty} \frac{1}{a_{\rho}(T)} \log \mathbb{P}\left(\sup _{t \in[r T, T]}\{Z(t)\}<0\right)<0,
\end{aligned}
$$

from which the LHS of (1.13) trivially follows. As for the RHS of (1.13), the derivation of (2.20) remains valid here, leading to

$$
\liminf _{T \rightarrow \infty} \frac{1}{\widehat{a}_{\rho}(T)} \log P\left(\sup _{t \in[0, T]}\{Z(t)\}<0\right)>-\infty,
$$

for $\widehat{a}_{\rho}(T)$ of (2.19). Since $T \mapsto \rho(T)$ is non-decreasing, the map $T \mapsto a_{\rho}(T)$ is differentiable a.e., with

$$
T I_{\rho}(T) \frac{d \log a_{\rho}(T)}{d T} \geq \int_{0}^{T}(\rho(x)-\rho(T)) d x .
$$

The RHS is eventually non-negative due to our assumption that the positive and eventually nonincreasing $\rho(T)$ decreases to zero as $T \rightarrow \infty$. Thus, the slowly varying $a_{\rho}(\cdot)$ is eventually nondecreasing, resulting for large enough $T_{1}$ with

$$
\widehat{a}_{\rho}(T)=\sum_{i=1}^{m} a_{\rho}\left(T_{i}\right) \leq m a_{\rho}\left(T_{m}\right) \lesssim a_{\rho}(T) \log T
$$

thereby completing the proof.

Proof of Lemma 2.8. (a). Recall that $I_{\rho}(b)-I_{\rho}(a)=\int_{a}^{b} x^{-\alpha} L_{\rho}(x) d x$. Let $\delta:=a / b$ and $\mu_{\alpha}$ denote the probability measure on $[0,1]$ of density $(1-\alpha) y^{-\alpha}$. The change of variable $x=y b$ transforms our claim (2.1) to

$$
\lim _{b \rightarrow \infty} \sup _{\delta \in[0,1)}\left|\frac{\int_{\delta}^{1}\left(\frac{L_{\rho}(y b)}{L_{\rho}(b)}-1\right) d \mu_{\alpha}(y)}{\mu_{\alpha}([\delta, 1])}\right|=0 .
$$


For bounded below $\delta>0$ this follows from the uniformity of the convergence $L_{\rho}(y b) / L_{\rho}(b) \rightarrow 1$, w.r.t. $y$ in a compact subset of $(0,1]$ (see Remark 2.7). Further, $\mu_{\alpha}([0, \delta])=\delta^{1-\alpha} \rightarrow 0$ as $\delta \rightarrow 0$, so fixing $0<\eta<1-\alpha$, it suffices to show that for some $b_{0}$ and $\kappa$ finite, all $b \geq b_{0}$ and any $\delta \in(0,1]$,

$$
\int_{0}^{\delta} \frac{L_{\rho}(y b)}{L_{\rho}(b)} d \mu_{\alpha}(y) \leq \kappa \delta^{1-\alpha-\eta}
$$

Indeed, recall Remark 2.7 on existence of $K$ finite, such that $L_{\rho}(y b) \leq y^{-\eta} L_{\rho}(b)$ whenever $b \geq y b \geq$ $K$. Hence, with $\int_{0}^{\delta} y^{-\eta} d \mu_{\alpha}(y)=c \delta^{1-\alpha-\eta}$ for some $c=c(\alpha, \eta)$ finite, we only need to consider the contribution of $y \leq \delta \wedge K / b$ to the LHS of (2.37). Since $\rho \in \mathcal{R}_{\alpha}$ is $(0,1]$-valued, the latter is at most $(\delta \wedge K / b) / \rho(b)$ which for $b \geq b_{0}$ is further bounded by $\delta(1 \wedge K /(\delta b)) b^{\alpha+\eta}$, so the elementary inequality $(1 \wedge x) \leq x^{\alpha+\eta}$ yields (2.37).

(b). For $\alpha>1$ taking as $\mu_{\alpha}$ the probability measure on $[1, \infty)$ of density $(\alpha-1) y^{-\alpha}$, the same change of variable as in part (a), transforms (2.2) into

$$
\lim _{b \rightarrow \infty} \sup _{\delta \in(1, \infty)}\left|\frac{\int_{1}^{\delta}\left(\frac{L_{\rho}(y b)}{L_{\rho}(b)}-1\right) d \mu_{\alpha}(y)}{\mu_{\alpha}([1, \delta])}\right|=0 .
$$

As in part (a), for bounded above $\delta$ this trivially follows from the uniform convergence $L_{\rho}(y b) / L_{\rho}(b) \rightarrow$ 1 , and since $\mu_{\alpha}([\delta, \infty))=\delta^{1-\alpha} \rightarrow 0$ as $\delta \rightarrow \infty$, it suffices to show that

$$
\lim _{\delta \uparrow \infty} \limsup _{b \rightarrow \infty} \int_{\delta}^{\infty} \frac{L_{\rho}(y b)}{L_{\rho}(b)} d \mu_{\alpha}(y)=0 .
$$

To this end, we fix $0<\eta<\alpha-1$ and recall that $L_{\rho}(y b) \leq y^{\eta} L_{\rho}(b)$ whenever $y b \geq b \geq K$. Since $\int_{\delta}^{\infty} y^{\eta} d \mu_{\alpha}(y) \rightarrow 0$ for $\delta \rightarrow \infty$, this completes the proof of (2.38) and of the lemma.

Proof of Lemma [2.9. (a). In case $\alpha \in[0,1)$ it follows by (2.1) (for $a=0$ ), that

$$
\lim _{b \rightarrow \infty} \frac{I_{\rho}(b)}{b \rho(b)}=\frac{1}{1-\alpha}
$$

and consequently $I_{\rho}(\cdot)$ is regularly varying of order $1-\alpha$. Turning to show that the increasing function $I_{\rho}(\cdot)$ is slowly varying when $\alpha=1$, it suffices to show that for any $\lambda>1$,

$$
\limsup _{T \rightarrow \infty}\left\{\frac{I_{\rho}(\lambda T)-I_{\rho}(T)}{I_{\rho}(T)}\right\} \leq 0 .
$$

To this end, fixing $\delta \in(0,1)$, we have that

$$
I_{\rho}(T) \geq \int_{\delta T}^{T} \frac{L_{\rho}(x)}{x} d x \geq \log (1 / \delta) \inf _{x \in[\delta T, T]}\left\{L_{\rho}(x)\right\}
$$

whereas

$$
I_{\rho}(\lambda T)-I_{\rho}(T)=\int_{T}^{\lambda T} \frac{L_{\rho}(x)}{x} d x \leq \log \lambda \sup _{x \in[T, \lambda T]}\left\{L_{\rho}(x)\right\} .
$$

Dividing (2.42) by (2.41) and taking $T \rightarrow \infty$, we arrive at the bound

$$
\limsup _{T \rightarrow \infty}\left\{\frac{I_{\rho}(\lambda T)-I_{\rho}(T)}{I_{\rho}(T)}\right\} \leq \frac{\log \lambda}{\log (1 / \delta)} .
$$

Taking now $\delta \rightarrow 0$ yields (2.40) and thereby that $I_{\rho}(\cdot)$ is slowly varying. Finally, since $I_{\rho}(\infty)<\infty$ when $\alpha>1$, the function $I_{\rho}(\cdot)$ is then (trivially) slowly varying at $\infty$. 
Proceeding to establish (2.3), by the regular variation of $\rho(\cdot)$ we have that for any $M>0$,

$$
\sup _{\ell \geq 1}\left\{\frac{M \rho(\ell M)}{\int_{\ell M}^{(\ell+1) M} \rho(t) d t}\right\} \leq \sup _{x \geq M} \sup _{\theta \in[1,2]}\left\{\frac{\rho(x)}{\rho(\theta x)}\right\}=: \kappa(M),
$$

with $\kappa(\cdot)$ non-increasing, hence uniformly bounded by universal $\kappa_{\star}$ finite (on some $\left[M_{0}, \infty\right)$ ). Consequently, for any $M \geq M_{0}$ and $n \geq 1$,

$$
M \sum_{\ell=1}^{n} \rho(\ell M) \leq \kappa_{\star}\left[I_{\rho}((n+1) M)-I_{\rho}(M)\right],
$$

and (2.3) follows by the regular variation of $I_{\rho}(\cdot)$. If $I_{\rho}(\infty)<\infty$, the RHS of (2.43) goes to zero when $n \rightarrow \infty$ followed by $M \rightarrow \infty$, thus yielding (2.4).

(b). Fixing $\epsilon>0$, by the regular variation of $\rho$ we have that for any $M \geq M_{\epsilon}$ and $\ell \geq K_{\epsilon}$

$$
\left|\frac{\int_{(\ell-1) M}^{\ell M} \rho(t) d t}{M \rho(\ell M)}-1\right| \leq \sup _{\lambda \in[1-1 / \ell, 1]}\left|\frac{\rho(\lambda \ell M)}{\rho(\ell M)}-1\right| \leq \epsilon .
$$

Thus, setting $n:=\lceil T / M\rceil$ we have for any $M \geq M_{\epsilon}$,

$$
I_{\rho}(T) \leq \sum_{\ell=1}^{n} \int_{(\ell-1) M}^{\ell M} \rho(t) d t \leq I_{\rho}\left(K_{\epsilon} M\right)+(1+\epsilon) M \sum_{\ell=1}^{n} \rho(\ell M) .
$$

If $\alpha \in(0,1]$ then the regularly varying $I_{\rho}(\cdot)$ has order $1-\alpha<1$, hence $I_{\rho}(K M) / M \rightarrow 0$ when $M \rightarrow \infty$ and $K$ is fixed. From (2.39) the same holds even for $\alpha=0$, provided $\rho(x) \rightarrow 0$. Note that when $\alpha<1$ necessarily $I_{\rho}(\cdot)$ diverges, and our hypothesis extends this conclusion to the case of $\alpha=1$. Thus, fixing $\mu>0$ we have that $M(T)=\mu I_{\rho}(T) \rightarrow \infty$ when $T \rightarrow \infty$. In particular, dividing both sides of (2.44) by $M=\mu I_{\rho}(T)$, then taking $T \rightarrow \infty$, yields

$$
\frac{1}{\mu} \leq(1+\epsilon) \liminf _{T \rightarrow \infty} \sum_{\ell=1}^{n} \rho(\ell M) \text {. }
$$

Taking now $\epsilon \downarrow 0$ establishes the lower bound of (2.5). The same reasoning we have used in deriving (2.44), leads also to

$$
I_{\rho}(n M) \geq I_{\rho}\left(K_{\epsilon} M\right)+(1-\epsilon) M \sum_{\ell=K_{\epsilon}+1}^{n} \rho(\ell M) .
$$

We divide both sides by $M=\mu I_{\rho}(T)$, then take $T \rightarrow \infty$ followed by $\epsilon \downarrow 0$. This in turn results with the corresponding upper bound of (2.5), since by (2.43), upon fixing $K<\infty$,

$$
\limsup _{M \rightarrow \infty} \sum_{\ell=1}^{K} \rho(\ell M) \leq \kappa_{\star} \limsup _{M \rightarrow \infty} \frac{I_{\rho}((K+1) M)}{M}=0,
$$

while by (2.39), $n \geq \mu^{-1} T / I_{\rho}(T) \rightarrow \infty$ and hence

$$
1 \leq \frac{I_{\rho}(n M)}{I_{\rho}(T)} \leq \frac{I_{\rho}(n M)}{I_{\rho}((n-1) M)}
$$

where the RHS converges to 1 when $T \rightarrow \infty$, due to the regular variation of $I_{\rho}(\cdot)$. 
Proof of Lemma 2.10. By (1.7) we have the existence of $C<\infty$ and $\eta \in(0,1)$ such that for any $\tau_{0}$ large enough,

$$
\sup _{\tau \geq \tau_{0}} \sup _{t \geq \tau / \eta} A(t, t+\tau) \leq C \rho\left(\tau_{0}\right) .
$$

Fixing $\epsilon>0$ we take $\tau_{0}$ large enough to assure that $C \rho\left(\tau_{0}\right) \leq \epsilon$ (which is always possible since $\rho(\tau) \rightarrow 0$ when $\tau \rightarrow \infty)$. For such $\tau_{0}$ and $n:=\left\lceil M / \tau_{0}\right\rceil$ we set

$$
t_{i}:=s+(i-1) \tau_{0} \in[s, s+M], \quad i=1, \ldots, n,
$$

noting that if $s \geq M / \eta$ then for any $1 \leq i, j \leq n$,

$$
t_{i} \geq s \geq \frac{M}{\eta} \geq \frac{(n-1) \tau_{0}}{\eta} \geq \frac{\left|t_{i}-t_{j}\right|}{\eta}
$$

and consequently, by (2.46)

$$
\mathbb{E}\left[Z\left(t_{i}\right) Z\left(t_{j}\right)\right]=A\left(t_{i}, t_{j}\right) \leq C \rho\left(\tau_{0}\right) \leq \epsilon .
$$

By Slepian's lemma and the union bound, we then have for i.i.d. standard normal $\left\{X_{i}\right\}_{i=0}^{n}$, any $r \in \mathbb{R}, s \geq M / \eta$ and $\epsilon<5 / 9$,

$$
\begin{aligned}
\mathbb{P}\left(\sup _{t \in[s, s+M]}\{Z(t)\}<r\right) & \leq \mathbb{P}\left(\sup _{i=1}^{n}\left\{Z\left(t_{i}\right)\right\}<r\right) \leq \mathbb{P}\left(\sup _{i=1}^{n}\left\{\sqrt{1-\epsilon} X_{i}+\sqrt{\epsilon} X_{0}\right\}<r\right) \\
& \leq \mathbb{P}\left(X_{0}<-r \epsilon^{-1 / 2}\right)+\mathbb{P}\left(X_{1}<3 r\right)^{n} .
\end{aligned}
$$

Setting $r=\sqrt{\delta \log M}$ we note that for $\delta<0.1$ and all $M$ large enough

$$
\mathbb{P}\left(X_{1}<3 r\right)^{n} \leq e^{-n \mathbb{P}\left(X_{1} \geq 3 r\right)} \leq e^{-\sqrt{M}} .
$$

Thus, from (2.47) we deduce that

$$
\limsup _{M \rightarrow \infty} \frac{1}{\log M} \sup _{s \geq M / \eta} \log \mathbb{P}\left(\sup _{t \in[s, s+M]}\{Z(t)\}<\sqrt{\delta \log M}\right) \leq-\frac{\delta}{2 \epsilon}
$$

and taking $\epsilon \downarrow 0$ results with the desired conclusion (2.6).

\section{Proof of Theorem 1.8}

Part (c) of Theorem 1.8 relies on Theorem 1.2 whereas parts (a) and (b) follow from [DM, Theorem 1.6]. For the latter task we extend the scope of [DM, Lemma 1.8] to non-stationary $A_{k}(\cdot, \cdot)$ and, for fully handling the $\gamma=2$ case, relax the uniform correlation tail decay requirement of [DM, (1.15)].

Lemma 3.1. Suppose $\left\{Z_{t}^{(k)}\right\}$, for $1 \leq k \leq \infty$, are centered Gaussian processes on $[0, \infty)$, of nonnegative covariance $A_{k}$ normalized to have $A_{k}(s, s)=1$ for all $s \geq 0$, such that $Z_{t}^{(\infty)}$ is a stationary process and $A_{k}(s, s+\tau) \rightarrow A_{\infty}(0, \tau)$ when $k \rightarrow \infty$, uniformly in $s \geq 0$. Suppose

$$
\limsup _{k, \tau \rightarrow \infty} \sup _{s \geq 0}\left\{\frac{A_{k}(s, s+\tau)}{\widetilde{\rho}(\tau)}\right\}<\infty
$$

for some integrable $\widetilde{\rho} \in \mathcal{R}_{\alpha}, \alpha \geq 1$, and in addition $A_{\infty}(0, \tau)$ is non-increasing, such that

$$
a_{h, \theta}^{2}:=\inf _{0<t \leq h}\left\{\frac{A_{\infty}(0, \theta t)-A_{\infty}(0, t)}{1-A_{\infty}(0, t)}\right\}>0,
$$


and there exists $\eta>1$ such that

$$
\limsup _{u \downarrow 0}|\log u|^{\eta} \sup _{1 \leq k \leq \infty, s \geq 0, \tau \in[0, u]}\left(1-A_{k}(s, s+\tau)\right)<\infty .
$$

Then we have

$$
-\lim _{k, T \rightarrow \infty} \frac{1}{T} \log \mathbb{P}\left(\sup _{t \in[0, T]}\left\{Z_{t}^{(k)}\right\}<0\right)=b\left(A_{\infty}\right) .
$$

Proof. The statement (3.4) is shown in [DM, Theorem 1.6] to hold under the following assumptions:

$$
\begin{gathered}
\limsup _{k, \tau \rightarrow \infty} \sup _{s \geq 0}\left\{\frac{\log A_{k}(s, s+\tau)}{\log \tau}\right\}<-1, \\
-\limsup _{M \rightarrow \infty} \frac{1}{M} \log \mathbb{P}\left(\sup _{t \in[0, M]} Z_{t}^{(\infty)}<M^{-\eta}\right)=b\left(A_{\infty}\right) \text { for all } \eta>0,
\end{gathered}
$$

and there exists $\zeta>0, M_{1}<\infty$ such that for any $z \in[0, \zeta]$ we have

$$
\begin{aligned}
\mathbb{P}\left(\sup _{t \in[0, M]} Z_{t}^{(\infty)}<z\right) & \leq \liminf _{k \rightarrow \infty} \inf _{s \geq 0} \mathbb{P}\left(\inf _{t \in[0, M]} Z_{s+t}^{(k)}<z\right) \\
& \leq \limsup _{k \rightarrow \infty} \sup _{s \geq 0} \mathbb{P}\left(\inf _{t \in[0, M]} Z_{s+t}^{(k)}<z\right) \leq \mathbb{P}\left(\sup _{t \in[0, M]} Z_{t}^{(\infty)} \leq z\right) .
\end{aligned}
$$

We verify that both (3.6) and (3.7) hold here, then adapt the proof of [DM, Theorem 1.6] to apply also when $\alpha=1$ in (3.1) (while (3.5) follows from (2.19) if $\alpha>1$ ).

It follows from the proof of [DM, Lemma 1.8], that (3.3) yields the a.s. continuity of $s \mapsto Z_{s}^{(k)}$ for $1 \leq k \leq \infty$, and that for any $M<\infty$, the collection $\left\{Z_{s+}^{(k)}, k \in \mathbb{N}, s \geq 0\right\}$ is uniformly tight in the space $\mathcal{C}[0, M]$ of continuous functions on $[0, M]$, equipped with the topology of uniform convergence. This and $A_{k}(s, s+\cdot) \rightarrow A_{\infty}(0, \cdot)$ uniformly in $s$, result with (3.7). Indeed, the failure of (3.7) amounts to having $M<\infty, z \in \mathbb{R}, \epsilon>0, k_{n} \uparrow \infty$ and $s_{n} \geq 0$, such that either

$$
\begin{aligned}
& \quad \inf _{n} \mathbb{P}\left(\sup _{t \in[0, M]}\left\{Z_{s_{n}+t}^{\left(k_{n}\right)}\right\}<z\right) \geq \mathbb{P}\left(\sup _{t \in[0, M]}\left\{Z_{t}^{(\infty)}\right\} \leq z\right)+\epsilon, \\
& \text { or } \\
& \quad \sup _{n} \mathbb{P}\left(\sup _{t \in[0, M]}\left\{Z_{s_{n}+t}^{\left(k_{n}\right)}\right\}<z\right) \leq \mathbb{P}\left(\sup _{t \in[0, M]}\left\{Z_{t}^{(\infty)}\right\}<z\right)-\epsilon .
\end{aligned}
$$

Since $A_{k_{n}}\left(s_{n}, s_{n}+\cdot\right) \rightarrow A_{\infty}(0, \cdot)$, all f.d.d.-s of the Gaussian processes $\left\{Z_{s_{n}+}^{\left(k_{n}\right)}\right\}$ converge to those of $Z^{(\infty)}$. Thus $Z^{(\infty)}$ is the limit in distribution on $\mathcal{C}[0, M]$ of $\left\{Z_{s_{n}+}^{\left(k_{n}\right)}\right\}$ and necessarily

$$
\sup _{t \in[0, M]}\left\{Z_{s_{n}+t}^{\left(k_{n}\right)}\right\} \stackrel{d}{\rightarrow} \sup _{t \in[0, M]}\left\{Z_{t}^{(\infty)}\right\}
$$

in contradiction with (3.8).

Next, recall [LS, Theorem 3.1(iii)], that for non-increasing $\tau \mapsto A_{\infty}(0, \tau)$, (3.6) yields the continuity of $\varepsilon \mapsto b\left(A_{\infty} ; \varepsilon\right)$, where

$$
b\left(A_{\infty}, \varepsilon\right):=-\lim _{T \rightarrow \infty} \frac{1}{T} \log \mathbb{P}\left(\sup _{t \in[0, T]} Z_{t}^{(\infty)}<\varepsilon\right)
$$

exists by Slepian's lemma, which in particular verifies the weaker condition (3.6) as well.

It thus remains to modify the proof of [DM, Theorem 1.6] to work under the assumption (3.1) instead of (3.5). Since the lower bound of [DM, Theorem 1.6] does not involve [DM, (1.15)] it 
suffices to adapt the proof of the matching upper bound. To this end, by (3.1) and the regular variation of $\widetilde{\rho}$, there exist $k_{0}, \tau_{0}$ and $C$ finite such that $\widetilde{\rho}$ is non-increasing on $\left[\tau_{0}, \infty\right)$ and

$$
A_{k}(s, t) \leq C \widetilde{\rho}(|s-t|), \quad \forall k \geq k_{0}, s \geq 0,|t-s| \geq \tau_{0} .
$$

Fixing $\delta>0$ and $M \geq \tau_{0} / \delta$, consider, as in [DM, proof of Theorem 1.6], a maximal collection $\mathcal{J}_{T}$ of $N$ intervals $I_{i} \subset[0, T]$ of length $M$ each, which are $\delta M$-separated. Then, setting

$$
\gamma=\gamma(\delta M):=4 C \sum_{i=1}^{\infty} \widetilde{\rho}(i \delta M)
$$

and the symmetric $N$-dimensional matrix $\mathbf{B}=\{B(i, j)\}$ with $B(i, i)=1$ and otherwise $B(i, j)=$ $\frac{C}{\gamma} \widetilde{\rho}(|i-j| \delta M)$ non-increasing in $|i-j|$, we have that

$$
\max _{1 \leq i \leq N} \sum_{j \neq i} B(i, j) \leq \frac{2 C}{\gamma} \sum_{i=1}^{\infty} \widetilde{\rho}(i \delta M) \leq \frac{1}{2} .
$$

Hence, all eigenvalues of $\mathbf{B}$ lie within [1/2,3/2]. Further, if $s \in I_{i}$ and $t \in I_{j}$, then $A_{k}(s, t) \leq \gamma B(i, j)$ by (3.10) and the monotonicity of $\widetilde{\rho}$. Consequently, the relation of [DM, (2.3)] holds for any $s, t \in \mathcal{J}_{T}$. The latter allows us to proceed along the derivation of [DM. (2.4)-(2.6)], except for replacing the terms $\gamma^{\delta}$, from the RHS of [DM, (2.4)] onward, by $\varepsilon>0$ (independent of $\gamma$ ). We thus deduce the following variant of [DM, (2.6)],

$$
\begin{aligned}
\limsup _{k, T \rightarrow \infty} \frac{1}{T} \log \mathbb{P} & \left(\sup _{t \in[0, T]}\left\{Z_{t}^{(k)}\right\}<0\right) \\
& \leq \frac{1}{M(1+\delta)} \log \left[\mathbb{P}\left(\sup _{t \in[0, M]}\left\{Z_{t}^{(\infty)}\right\}<3 \varepsilon\right)+\sqrt{3} \mathbb{P}\left(X_{1} \geq \sqrt{2 / 3} \varepsilon \gamma^{-1 / 2}\right)\right] .
\end{aligned}
$$

Here $X_{1}$ is standard normal and $M \gamma(\delta M) \rightarrow 0$ when $M \rightarrow \infty$ (by (2.4) and (3.11)), hence

$$
\limsup _{M \rightarrow \infty} \frac{1}{M} \log \mathbb{P}\left(X_{1} \geq \sqrt{2 / 3} \varepsilon \gamma^{-1 / 2}\right) \leq-\frac{\varepsilon^{2}}{6} \liminf _{M \rightarrow \infty}(M \gamma)^{-1}=-\infty,
$$

so in the limit $M \rightarrow \infty$ the RHS of (3.12) is at most $-b\left(A_{\infty} ; 3 \varepsilon\right) /(1+\delta)$, for $b\left(A_{\infty} ; \cdot\right)$ of (3.9). Thus, considering $\varepsilon, \delta \downarrow 0$ yields the upper bound of [DM, Theorem 1.6].

Finally, from Lemma 2.6 the events $\left\{\sup _{t \in[0, M]}\left\{Z_{t}^{(\infty)}\right\}<0\right\}$ have positive probability, hence $b\left(A_{\infty} ; 0\right)$ is finite (by the non-negativity of $A_{\infty}$ and Slepian's lemma). Further, in view of (3.13) the RHS of (3.12) is strictly negative for $M$ large enough, hence its LHS, namely $-b\left(A_{\infty} ; 0\right)$ is also strictly negative.

Proof of part (e) of Corollary 1.10: This is a direct application of Lemma 3.1. Indeed, the relation between occupation times of $\mathbf{0}$ by $S_{u}^{(q)}$, and the number of returns to $\mathbf{0}$ by the corresponding embedded discrete time random walk, implies that

$$
I_{\rho^{(q)}}(\infty)-I_{\rho^{(q)}}(\tau)=\mathbb{E}\left[G_{N_{\tau}}^{(q)}\right]
$$

for a unit rate Poisson process $\left\{N_{\tau}\right\}$. Thus, here the auto-correlation of (1.18) is

$$
\bar{C}_{\rho^{(q)}}(0, \tau)=\frac{1}{G_{0}^{(q)}} \mathbb{E}\left[G_{N_{\tau}}^{(q)}\right] .
$$


Clearly, $G_{0}^{(q)}-1=G_{1}^{(q)} \geq G_{k}^{(q)} \geq G_{k+1}^{(q)}$ for all $k \geq 1$, resulting with the bounds

$$
1-\mathbb{P}\left(N_{\tau} \geq 1\right) \leq \bar{C}_{\rho^{(q)}}(0, \tau) \leq 1-\frac{1}{G_{0}^{(q)}} \mathbb{P}\left(N_{\tau} \geq 1\right),
$$

so by the assumed convergence to one of $G_{0}^{\left(q_{d}\right)}$ we have that

$$
\lim _{d \rightarrow \infty} \bar{C}_{\rho^{\left(q_{d}\right)}}(0, \tau)=1-\mathbb{P}\left(N_{\tau} \geq 1\right)=e^{-\tau}
$$

Recall that the stationary Ornstein-Uhlenbeck (OU) process has persistence exponent $b=1$, continuous sample path and the correlation function $e^{-|\tau|}$ for which holds. In addition, from the uniform lower bound on the LHS of (3.15),

$$
\lim _{u \rightarrow 0}|\log u|^{2} \sup _{d}\left(1-\bar{C}_{\rho^{\left(q_{d}\right)}}(0, u)\right)=0,
$$

so (3.3) holds as well. Finally, from (3.14)

$$
\bar{C}_{\rho^{\left(q_{d}\right)}}(0, \tau) \leq \mathbb{P}\left(N_{\tau} \leq \tau / 2\right)+G_{\tau / 2}^{\left(q_{d}\right)},
$$

hence (3.1) follows from the assumed uniform tail bound $G_{\tau / 2}^{\left(q_{d}\right)} \leq \kappa \tau^{-2}$.

Equipped with Lemma 3.1 we proceed to establish Theorem 1.8 ,

Proof of Theorem 1.8 .

(a). To prove (1.20), we apply Lemma 3.1 for the normalized, centered Gaussian processes $\left\{Z_{t}^{(k)}\right\}$, $1 \leq k \leq \infty$, of correlation functions

$$
A_{k}(s, t):=C_{\rho}(s+k, t+k), \quad A_{\infty}(s, t):=\bar{C}_{\rho}(s, t), \quad s, t \geq 0 .
$$

Specifically, from (1.18) we see that $A_{\infty}(0, \tau)$ is non-increasing and $A_{k}(s, s+\tau) \rightarrow A_{\infty}(0, \tau)$ as $k \rightarrow \infty$, uniformly in $s \geq 0$. Further, with $\rho \in \mathcal{R}_{\gamma}$ uniformly bounded and $I_{\rho}(\cdot)$ strictly positive, non-decreasing, it follows from (1.17) that for $s \geq 1, \tau>0$,

$$
1-C_{\rho}(s, s+\tau) \leq \frac{I_{\rho}(2 s+2 \tau)-I_{\rho}(2 s+\tau)+I_{\rho}(\tau)}{I_{\rho}(2 s)} \leq \frac{2 \sup _{x \geq 0}\{\rho(x)\}}{I_{\rho}(2)} \tau .
$$

Thus $t \mapsto Y_{\rho}(t)$ is a.s. continuous on $[1, \infty)$ and with the preceding holding for $\bar{C}_{\rho}(\cdot)$, so does (3.3). Since $C_{\rho}(\cdot)$ is non-negative, by Slepian's lemma we have that for any $k \in(1, T)$,

$$
\mathbb{P}\left(\sup _{t \in[k, T+k]}\left\{Y_{\rho}(t)\right\}<0\right) \geq \mathbb{P}\left(\sup _{t \in[1, T+k]}\left\{Y_{\rho}(t)\right\}<0\right) \geq \mathbb{P}\left(\sup _{t \in[1, k]}\left\{Y_{\rho}(t)\right\}<0\right) \mathbb{P}\left(\sup _{t \in[k, T+k]}\left\{Y_{\rho}(t)\right\}<0\right)
$$

and with $Y_{\rho}(t)$ continuous the first term on the RHS is strictly positive (see Lemma 2.6). It thus suffices to confirm that

$$
-\lim _{k \rightarrow \infty} \lim _{T \rightarrow \infty} \frac{1}{T} \log \mathbb{P}\left(\sup _{t \in[k, T+k]}\left\{Y_{\rho}(t)\right\}<0\right)=b\left(\bar{C}_{\rho}\right) .
$$

With $Z_{t}^{(k)}=Y_{\rho}(k+t)$, the identity (3.16) is merely (3.4). We thus complete the proof upon verifying the remaining two assumptions of Lemma 3.1, first showing that (3.1) holds for the integrable $\widetilde{\rho}(s)=s \rho(s)$, then establishing the positivity of $a_{h, \theta}^{2}\left(\bar{C}_{\rho}\right)$ of (3.2). Turning to the first task, setting $s_{k}=2 s+2 k \geq 2$ note that for any $s \geq 0$,

$$
A_{k}(s, s+\tau)=\frac{I_{\rho}\left(s_{k}+\tau\right)-I_{\rho}(\tau)}{\sqrt{I_{\rho}\left(s_{k}+2 \tau\right) I_{\rho}\left(s_{k}\right)}} \leq \frac{I_{\rho}(\infty)-I_{\rho}(\tau)}{I_{\rho}(2)},
$$


out of which we get (3.1), since by (2.2) (for $a \uparrow \infty$ and $\alpha=\gamma>1$ ),

$$
\frac{I_{\rho}(\infty)-I_{\rho}(\tau)}{\tau \rho(\tau)} \rightarrow \frac{1}{\gamma-1}<\infty
$$

Next, setting $g(\theta, \tau):=\int_{\theta}^{1} \rho(\tau y) d y$ we have from (1.18) that

$$
a_{h, \theta}^{2}\left(\bar{C}_{\rho}\right)=\inf _{0<\tau<h}\left\{\frac{g(\theta, \tau)}{g(0, \tau)}\right\} \geq(1-\theta) \frac{\inf _{x \in[0, h]} \rho(x)}{\sup _{x \in[0, h]} \rho(x)}>0,
$$

by our hypothesis that $\rho \in \mathcal{R}_{\gamma}$ is uniformly bounded away from zero on compacts.

(b). Considering the Lamperti transformation $t=e^{v}$ on $[0, V]$ (where $T=e^{V}$ ), similarly to part (a), due to Lemma 2.6 and the sample path continuity of $Y_{\rho}(\cdot)$ we establish (1.21) upon showing that

$$
-\lim _{k \rightarrow \infty} \lim _{V \rightarrow \infty} \frac{1}{V} \log \mathbb{P}\left(\sup _{v \in[0, V]}\left\{Y_{\rho}\left(e^{v+k}\right)\right\}<0\right)=b\left(C_{\gamma}^{\star}\right) \in(0, \infty) .
$$

The identity (3.19) is merely (23.4) for the centered Gaussian processes $Z_{v}^{(k)}=Y_{\rho}\left(e^{v+k}\right)$ of correlation functions

$$
A_{k}(v, u):=C_{\rho}\left(e^{v+k}, e^{u+k}\right), \quad A_{\infty}(v, u):=C_{\gamma}^{\star}(v, u), \quad v, u \geq 0 .
$$

Thus, (3.19) follows once we verify all the assumptions of Lemma 3.1, at least for all $k \geq k_{0}$ finite. To this effect, for $\gamma \in[0,1)$ we have from (1.19) that $A_{k}(v, v+\tau) \rightarrow A_{\infty}(0, \tau)$, uniformly over $v \geq 0$, with $A_{\infty}(0, \tau)$ non-increasing. Further, for any $\theta \in(0,1)$, setting $g(0)=1-\theta^{1-\gamma}>0$ makes

$$
g(\tau):=\frac{C_{\gamma}^{\star}(0, \theta \tau)-C_{\gamma}^{\star}(0, \tau)}{1-C_{\gamma}^{\star}(0, \tau)},
$$

a continuous and strictly positive function on $[0, \infty)$. Thus, $a_{h, \theta}^{2}\left(C_{\gamma}^{\star}\right)$ being the infimum of $g(\tau)$ over $[0, h]$ must be positive, and so (3.2) holds.

Turning next to verify (3.1), setting $v_{k}:=e^{v+k} \geq e^{k}$ we have that

$$
A_{k}(v, v+\tau)=\frac{I_{\rho}\left(\left(e^{\tau}+1\right) v_{k}\right)-I_{\rho}\left(\left(e^{\tau}-1\right) v_{k}\right)}{\sqrt{I_{\rho}\left(2 v_{k}\right) I_{\rho}\left(2 e^{\tau} v_{k}\right)}}, \quad \forall v, \tau \geq 0 .
$$

Hence, three applications of (2.1) with $\alpha=\gamma$, for $b=\left(e^{\tau}+1\right) v_{k}, b=2 e^{\tau} v_{k}$ and $b=2 v_{k}$, yield that

$$
\lim _{k \rightarrow \infty} \sup _{v, \tau \geq 0}\left|R_{\rho}\left(v_{k}, \tau\right) \frac{A_{k}(v, v+\tau)}{A_{\infty}(0, \tau)}-1\right|=0
$$

where by the eventual monotonicity of $x^{ \pm 2 \eta} L_{\rho}(x)$ (see Remark 2.7), we further get that

$$
R_{\rho}(v, \tau):=\frac{\sqrt{L_{\rho}(2 v) L_{\rho}\left(2 e^{\tau} v\right)}}{L_{\rho}\left(\left(e^{\tau}+1\right) v\right)} \in\left(e^{-\tau \eta}, e^{\tau \eta}\right),
$$

for any $\eta>0, v \geq v_{0}(\eta)$ and all $\tau \geq 0$. Since $\gamma \mapsto C_{\gamma}^{\star}(0, \tau)$ is non-increasing, we have that for any $\gamma \in[0,1)$

$$
A_{\infty}(0, \tau) \leq C_{0}^{\star}(0, \tau)=e^{-|\tau| / 2}
$$

Combining this with (3.21) and (3.22) (say, for $\eta=1 / 4)$, we deduce that $A_{k}(v, v+\tau) \leq 2 e^{-\tau / 4}$ for any $k \geq k_{0}$ and all $v, \tau \geq 0$, which is more than enough for (3.1).

It thus remains to verify (3.3). To this effect, set $\xi:=\left(1-e^{-\tau}\right) / 2 \leq \tau$ and

$$
f(\xi ; b, \tau):=\frac{I_{\rho}(b)-I_{\rho}((1-\xi) b)+I_{\rho}(\xi b)}{I_{\rho}\left(e^{-\tau} b\right)} .
$$


Then, considering $b=2 e^{\tau} v_{k}$ in (3.20), we find that for any $v, \tau \geq 0$ and finite $k \geq 1$,

$$
1-A_{k}(v, v+\tau) \leq \sup _{b \geq 2 e^{k+\tau}}\{f(\xi ; b, \tau)\}
$$

As $1-C_{\gamma}^{\star}(0, \tau) \leq|\tau|^{1-\gamma}$ for $|\tau|$ small enough, we get (3.3) upon showing that for $\eta>0$ and $\kappa, b_{0}$ finite, $f(\xi ; b, \tau) \leq \kappa \xi^{1-\gamma-\eta}$, uniformly over $\tau \in[0,1]$ and $b \geq b_{0}$. To this end, setting

$$
F_{b}\left(a_{1}, a_{2}\right)=\int_{a_{1}}^{a_{2}} \frac{L_{\rho}(b y)}{L_{\rho}(b)} d \mu_{\gamma}(y)
$$

for $0 \leq a_{1} \leq a_{2} \leq 1$ and the measure $\mu_{\gamma}$ on $[0,1]$ of density $(1-\gamma) y^{-\gamma}$, recall (2.36) that

$$
\lim _{b \rightarrow \infty} \sup _{\delta \in[0,1)}\left|\frac{F_{b}(\delta, 1)}{1-\delta^{1-\gamma}}-1\right|=0
$$

and (2.37) that for some $b_{0}$ finite,

$$
\sup _{b \geq b_{0}} \sup _{\delta \in(0,1]}\left\{\frac{F_{b}(0, \delta)}{\delta^{1-\gamma-\eta}}\right\}<\infty .
$$

Further, we find as in the proof of Lemma 2.8(a), that

$$
f(\xi ; b, \tau)=\frac{F_{b}(1-\xi, 1)+F_{b}(0, \xi)}{F_{b}\left(0, e^{-\tau}\right)},
$$

where by the preceding, once $b$ is large enough $F_{b}(1-\xi, 1) \leq 2 \xi$ and $F_{b}(0, \xi) \leq \kappa \xi^{1-\gamma-\eta}$ for all $\xi$, while $F_{b}\left(0, e^{-\tau}\right) \geq F_{b}\left(0, e^{-1}\right)$ are bounded below away from zero.

(c). We get (1.22) upon applying Theorem 1.2 for the centered Gaussian process $Y_{\rho}(t), t \in[1, \infty)$ (with non-integrable $\widetilde{\rho}(s)=s \rho(s) \in \mathcal{R}_{\gamma-1}$ ). Turning to verify the three hypothesis of Theorem 1.2 , recall first that while proving part (a) we saw that $t \mapsto Y_{\rho}(t)$ is a.s. continuous and further showed that $\sup _{s \geq 1}\left(1-C_{\rho}(s, s+\tau)\right)$ decay fast enough in $\tau \rightarrow 0$ to imply that $\mathbb{E}\left[\sup _{t \in[0,1]}\left\{Y_{\rho}(s+t)\right\}\right]$ is uniformly bounded in $s \geq 1$. Next, similarly to (3.17),

$$
C_{\rho}(t, t+\tau) \leq \frac{I_{\rho}(\infty)-I_{\rho}(\tau)}{I_{\rho}(2)}
$$

and (1.7) follows from (3.18). Finally, if $\tau \in[0, \eta t]$ then by (2.2) with $\alpha=\gamma$, we deduce that for $\tau \rightarrow \infty$

$$
\frac{C_{\rho}(t, t+\tau)}{\widetilde{\rho}(\tau)} \geq \frac{I_{\rho}(h \tau)-I_{\rho}(\tau)}{\widetilde{\rho}(\tau) I_{\rho}(\infty)} \rightarrow \frac{1-h^{1-\gamma}}{(\gamma-1) I_{\rho}(\infty)},
$$

which since $h:=1+2 / \eta$ diverges with $\eta \downarrow 0$, yields (1.10) and thereby proves (1.22).

\section{REFERENCES}

[AT] Adler, R. J. and Taylor, J. E. Random fields and geometry. Springer, New York, 2007.

[AS] Aurzada, F. and Simon, T. Persistence probabilities and exponents. Lévy matters V, Lecture Notes in Math., 2149:183-221, Springer, 2015.

[BGT] Bingham, N. H., Goldie, C. M. and Teugels, J. L. Regular variation. Cambridge Univ. Press, Cambridge, 1987.

[BD] Bryc, W. L. and Dembo, A. On large deviations of empirical measures for stationary Gaussian processes, Stoch. Proc. Appl., 58:23-34, 1995.

[BMS] Bray, A.J., Majumdar, S.N. and Schehr, G. Persistence and first-passage properties in non-equilibrium systems, Advances in Physics, 62(3):225-361, 2013.

[DD] Dembo, A. and Deuschel, J.D. Aging for interacting diffusion processes, Ann. Inst. H. Poincare Probab. Statist. 43:461-480, 2007. 
[Deu] Deuschel, J.D. Invariance principle and empirical mean large deviations of the critical Ornstein-Uhlenbeck process, Ann. Prob. 17:74-90, 1989.

[Deu2] Deuschel, J.D. The random walk representation for interacting diffusion processes, Interacting stochastic systems, 377-393, Springer, Berlin, 2005.

[DM] Dembo, A, and Mukherjee, S. No zero-crossings for random polynomials and the heat equation, Ann. Probab., 43(1):85-118, 2015.

[DPSZ] Dembo, A., Poonen, B., Shao, Q. M. and Zeitouni, O. Random polynomials having few or no real zeros. J. Amer. Math. Soc., 15(4):857-892, 2002.

[DV] Donsker, M. D., and Varadhan, S. R. S. Large deviations for stationary Gaussian processes. Comm. Math. Phys., 97 1-2:187-210, 1985.

[FF] Feldheim, N. and Feldheim, O. Long gaps between sign-changes of Gaussian stationary processes. Int. Math. Res. Notices, 11:3021-3034, 2015.

[FS] Funaki, T. and Spohn, H., Motion by mean curvature from the Ginzburg-Landau interface model, Comm. Math. Phy., 185(1):1-36, 1997.

[Gar] Garet, O. Infinite dimensional dynamics associated to quadratic Hamiltonians, Markov Processes and Related Fields, 6:205-237, 2000.

[GOS] Giacomin, G., Olla, S. and Spohn, H., Equilibrium fluctuations for $\nabla \phi$ interface model, Ann. Probab., 29(3):1138-1172, 2001.

[GPS] Gambassi, A., Paul, R. and Schehr, G., Dynamic crossover in the persistence probability of manifolds at criticality, J. Stat. Mech., P12029, 2010.

[Ham] Hammersley, J.M., Harnesses, Proc. Fifth Berkeley Sympos. Mathematical Statistics and Probability (Berkeley, Calif., 1965/66), Vol. III: Physical Sciences, 89-117. Univ. California Press, Berkeley, Calif.

[KKMCBS] Krug, J., Kallabis, H., Majumdar, S.N.,Cornell, S.J., Bray, A.J. and Sire, C. Persistence exponents for fluctuating interfaces, Phys. Rev. E., 56(3):2702-2712, 1997.

[Lam] Lamperti, J. Semi-stable stochastic processes. Trans. Amer. Math. Soc. 104(1), 62-78, 1962

[LS] Li, W. V. and Shao, Q. M. Recent developments on lower tail probabilities for Gaussian processes. Cosmos, 1:95-106, 2005.

[Mol] Molchan, G. Survival exponents for some Gaussian processes. Int. J. of Stoch. Anal., 137271, 1-20, 2012.

[MB1] Majumdar, S.N. and Bray, A.J. Spatial persistence of fluctuating interfaces. Phys. Rev. Lett., 86(17):37003703, 2001.

[MB2] Majumdar, S.N. and Bray, A.J. Persistence of manifolds in non-equilibrium critical dynamics. Phys. Rev. Lett., 91(3):030602, 2003.

[Pic] Pickands, J., Asymptotic properties of maximum in a stationary Gaussian processes, Trans. Amer. Math. Soc., 145:75-86, 1969.

[NR] Newell, G.F. and Rosenblatt, M. Zero crossing probabilities for Gaussian stationary processes, Ann. Math. Statis., 33(4):1306-1313, 1962.

[Sak] Sakagawa, H. Persistence probability for a class of Gaussian processes related to random interface models, $A d v$. in Appl. Probab., 47(1):146-163, 2015.

[Shu] Shur, M.G., On the maximum of a Gaussian stationary process, Theor. Probability Appl., 10:354-357, 1965.

[Sle] Slepian, D. The one-sided barrier problem for Gaussian noise. Bell System Tech., 41:463-501, 1962.

[SM] Schehr, G. and Majumdar, S. N. Real roots of random polynomials and zero crossing properties of diffusion equation. J. of Stat. Phys., 132(2):235-273, 2008.

[UO] Uhlenbeck, G.E. and Ornstein, L.S. On the theory of Brownian motion. Phys. Rev. Lett., 36:823-841, 1930.

[Unt] Unterberger, J. Stochastic calculus for fractional Brownian motion with Hurst exponent $H>\frac{1}{4}$ : A rough path method by analytic extension, Ann. Probab., 37(2):565-614, 2009.

[LL] Lawler, G. F. and Limic, V., Random walk: a modern introduction, Cambridge Univ. Press, 2010.

[Wat] Watanabe, H., An asymptotic property of Gaussian stationary processes, Proc. Japan Acad. , 44:895-896, 1968.

*Department of Mathematics, Stanford University, Building 380, Sloan Hall, Stanford, CA 94305 , USA

Department of Statistics, Columbia University, 1011 SSW, 1255 Amsterdam Avenue, New York, Ny 10027, USA 